\title{
DIFFUSION ON A SPHERE WITH LOCALIZED TRAPS: MEAN FIRST PASSAGE TIME, EIGENVALUE ASYMPTOTICS, AND FEKETE POINTS*
}

\author{
DANIEL COOMBS ${ }^{\dagger}$, RONNY STRAUBE ${ }^{\ddagger}$, AND MICHAEL WARD $^{\dagger}$
}

\begin{abstract}
A common scenario in cellular signal transduction is that a diffusing surface-bound molecule must arrive at a localized signaling region on the cell membrane before the signaling cascade can be completed. The question then arises of how quickly such signaling molecules can arrive at newly formed signaling regions. Here, we attack this problem by calculating asymptotic results for the mean first passage time for a diffusing particle confined to the surface of a sphere, in the presence of $N$ partially absorbing traps of small radii. The rate at which the small diffusing molecule becomes captured by one of the traps is determined by asymptotically calculating the principal eigenvalue for the Laplace operator on the sphere with small localized traps. The asymptotic analysis relies on the method of matched asymptotic expansions, together with detailed properties of the Green's function for the Laplacian and the Helmholtz operators on the surface of the unit sphere. The asymptotic results compare favorably with full numerical results.
\end{abstract}

Key words. receptor clustering, matched asymptotic expansions, logarithmic expansions, Green's functions, mean first passage time, elliptic Fekete points

AMS subject classifications. 35B25, 35C20, 35P99, 92C05

DOI. $10.1137 / 080733280$

1. Introduction. In this paper we will consider the diffusion of molecules on the surface of a spherical cell and, in particular, study the rate of arrival of molecules to small absorbing traps on the cell surface. We are inspired by an array of biological situations in which a mobile cell-surface molecule undergoes a chemical reaction upon arriving at a localized region on the cell surface. By way of example, we now describe two situations in the activation of immune cells where the arrival of cell surface molecules at well-defined small regions is important to a cell signaling process.

Mast cells of the immune system present a particular receptor, named Fc $\epsilon$ RI, on their surface. When this receptor is bound to the common chain of an antibody ( $\operatorname{IgE}$ ), the cell is primed to respond to any antigen (for instance, pollen) that can subsequently bind to a number of antibody-linked receptors and hold them in close proximity [23]. This process forms a cluster of bound receptors and therefore leads, via a complex set of biochemical reactions, to cell activation and histamine release and ultimately the familiar symptoms of allergies. A key player in the signaling cascade is the transmembrane signaling protein LAT which must arrive at the cluster in order for signaling to be completed (see Figure 1(a)).

A very similar scenario plays out in experiments designed to examine the response of $T$ cells of the immune system to molecular stimuli (antigens) presented on synthetic surfaces. The $\mathrm{T}$ cells in the experiments present $\mathrm{T}$ cell receptors (TCRs) that can bind

\footnotetext{
* Received by the editors August 27, 2008; accepted for publication (in revised form) March 6, 2009; published electronically June 3, 2009.

http://www.siam.org/journals/siap/70-1/73328.html

${ }^{\dagger}$ Department of Mathematics, University of British Columbia, Vancouver V6T 1Z2, BC, Canada (coombs@math.ubc.ca, ward@math.ubc.ca). The research of these authors was supported by NSERC (Canada).

${ }^{\ddagger}$ Max-Planck Institute for Dynamics of Complex Technical Systems, 39106 Magdeburg, Germany (rstraube@mpi-magdeburg.mpg.de). This author's research was supported by the Ministry of Education of Saxony-Anhalt within the Research Center "Dynamic Systems."
}

302 
to the antigen molecules. Upon receptor binding, biochemical signaling pathways are activated that lead to $\mathrm{T}$ cell activation (see Figure 1(b)). In these experiments, the TCR distribution on the cell surface can be monitored using fluorescent tags, and the TCRs are observed to form submicrometer scale clusters that are believed to be critical for TCR signaling and antigen detection [45], [6], [50]. Again, TCR signaling is dependent on the presence of the transmembrane protein LAT within the TCR cluster (see Figure 1).
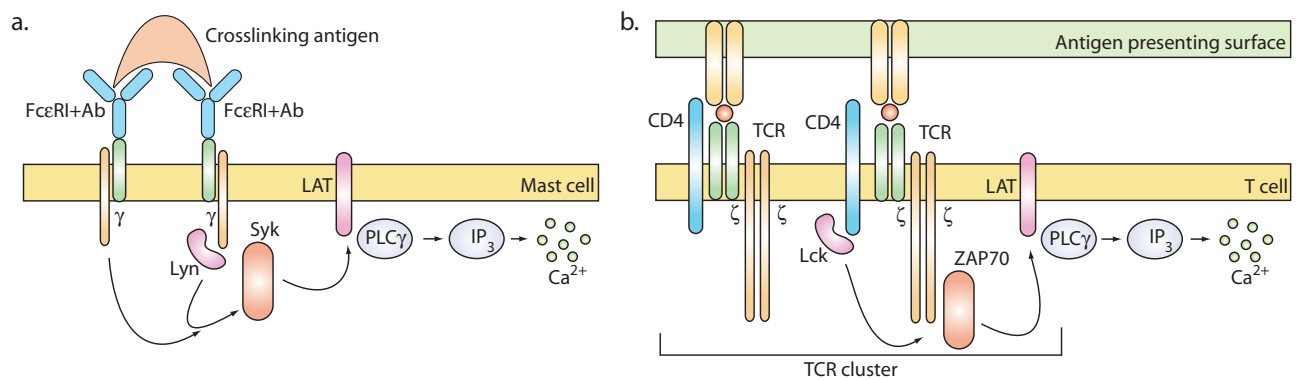

FIG. 1. Immune cell signaling involves the arrival of surface-bound signaling molecules to receptor clusters. (a) Multiple FcєRI receptors can be crosslinked by multivalent antigen, forming a cluster. This allows signaling to begin through the $\gamma$ chain of the receptor, and the signaling proteins Lyn and Syk. The signaling pathway shown here requires the surface-bound molecule LAT to be present at the receptor complex. (b) Binding by recognized antigen leads rapidly to TCR clustering and signaling through the proteins Lck and ZAP-70. The presence of LAT at the cluster is essential for the signaling process.

In both these examples, the creation of localized receptor clusters precedes the cell signaling cascade: a complex network of chemical reactions involving a large number of surface-bound and cytosolic signaling molecules. In order to understand how the cascade develops within each individual cluster, we would like to know how quickly essential signaling molecules can arrive. We would like to determine, for example, the rate of arrival of LAT to nascent receptor signaling clusters on $\mathrm{T}$ and mast cells of the immune system.

Inspired by these problems, where a mobile cell surface molecule must arrive at localized signaling domains to catalyze signal transduction and cell activation, we will focus on the general problem of the arrival of surface-bound signaling molecules to arbitrarily distributed small signaling clusters (henceforth, traps). We assume that the molecules are delivered to or activated at the cell surface with a space-dependent rate $M$ and, subsequently, perform free diffusion with an effective diffusion coefficient $D$. We formulate a mean first passage time problem for encounters on the surface of a spherical cell of radius $L$ with either completely, or partially, absorbing traps. The problem we consider can be viewed as one of many involving surface diffusion in the presence of traps.

In a cell-biological context, similar problems have been examined. A classic example is found in the phenomenon of receptor-mediated endocytosis [29], [19]. Cell surface receptors that bind extracellular ligands are found to reach the interior of the cell after interacting with coated pits (specialized membrane regions that contain clathrin). In our model, we can describe the coated pits as traps for diffusing surface receptors. Other biological examples include the delivery of proteins to dendritic spines in neuronal biology [1], [5], [36], [44] and the repeated binding of antigenic proteins in the immunological synapse [49], [12]. 
From a mathematical viewpoint, our singular perturbation problem on the surface of the sphere has a structure similar to that of the narrow escape problems analyzed in [20], [21], [36] (see also the references therein), involving the determination of the mean first passage time for a freely diffusing particle confined inside either a two- or a three-dimensional bounded domain that has absorbing windows of small measure on an otherwise reflecting boundary.

The outline of this paper is as follows. In section 2 we formulate the steadystate diffusion equation on the sphere with an inhomogeneous source term in the presence of localized traps of small radii. In addition, we formulate the eigenvalue problem governing the time-dependent long-time asymptotic approach to the steadystate problem. In section 2 we also highlight some previous results for the steady-state and eigenvalue problems, and in Appendix A we give some detailed analytical results for certain Green's functions that are central to the asymptotic analysis.

In section 3 we use the method of matched asymptotic expansions to calculate asymptotic solutions to the steady-state diffusion problem on the unit sphere with an inhomogeneous source term in the presence of $N$ partially absorbing traps of asymptotically small radii. The case of a constant spatially homogeneous source term pertains directly to the problem of calculating the mean first passage time on the unit sphere containing $N$ localized traps. By using the method of matched asymptotic expansions, the asymptotic solution is constructed in terms of the modified Green's function for the Laplacian on the unit sphere. This Green's function is well known from the analyses of vortex motion on the unit sphere (cf. [24], [25], and [26]).

In section 4 we asymptotically calculate the principal eigenvalue for the Laplacian on the unit sphere in the presence of $N$ traps on the surface of the sphere of asymptotically small radii. Similar problems in planar domains have been considered in [46] and [27], and on the boundary of the cylinder in [40]. For the case of perfectly absorbing traps of a common radius, we derive a two-term expansion for the principal eigenvalue in powers of $-1 / \log (\varepsilon a)$, where $\varepsilon a \ll 1$ is the common trap radius. It is shown analytically that the second term in the asymptotic expansion of the principal eigenvalue is maximized when the locations $x_{j}$ for $j=1, \ldots, N$ of the traps, with $\left|x_{j}\right|=1$, are chosen to maximize the discrete sum

$$
p\left(x_{1}, \ldots, x_{N}\right)=\sum_{i=1}^{N} \sum_{\substack{j=1 \\ j \neq i}}^{N} \log \left|x_{i}-x_{j}\right| .
$$

Such points are known as elliptic Fekete points (cf. [11], [39], [31], and [32]). In section 4 we also formulate a transcendental equation that effectively sums the infinite order expansion in powers of $-1 / \log (\varepsilon a)$ for the principal eigenvalue of the Laplacian in the presence of $N$ partially absorbing traps. This transcendental equation involves the Green's function for the Helmholtz operator on the unit sphere and its regular part, which are well known from the theory of high-frequency scattering (cf. [43], [38]).

In section 5 we favorably compare our asymptotic results for steady-state diffusion and the principal eigenvalue with full numerical results computed from COMSOL [8]. Finally, in section 6 we briefly discuss some possible extensions of the analysis presented herein to other, more intricate, models of biological localization phenomena on cell surfaces.

2. The surface diffusion equation and some previous results. We consider steady-state diffusion on the surface of the unit sphere $S$, with a spatially inhomogeneous source term $M$, in the presence of $N$ partially absorbing nonoverlapping traps. 
The dimensionless problem is formulated as

$$
\begin{gathered}
\triangle_{s} u=-M, \quad x \in S_{\varepsilon} \equiv S \backslash \cup_{j=1}^{N} \Omega_{\varepsilon_{j}}, \\
\varepsilon \nabla_{s} u \cdot \hat{n}+\kappa_{j}\left(u-b_{j}\right)=0, \quad x \in \partial \Omega_{\varepsilon_{j}} .
\end{gathered}
$$

The source term $M$ is assumed to be a smooth function on $S$ and independent of $u$. In (2.1b) the unit normal $\hat{n}$ points inwardly to the trap $\Omega_{\varepsilon_{j}}$. Each trap is centered at some $x_{j} \in S$ with $\left|x_{j}\right|=1$ and has radius $\mathcal{O}(\varepsilon)$, with $\varepsilon \ll 1$. The traps are assumed to be well separated in the sense that $\left|x_{i}-x_{j}\right|=\mathcal{O}(1)$ for $i, j=1, \ldots, N$. The constants $b_{j}$ in (2.1b) denote local threshold values in the sense that if $u>b_{j}$ $\left(u<b_{j}\right)$ near the $j$ th trap, then there will be a local flux of particles into (out of) that trap, which is proportional to the constant $\kappa_{j} / \varepsilon>0$. In $(2.1 \mathrm{~b})$, notice that $\varepsilon$ also has a dual role of setting the asymptotic range of the transfer (or Biot number) coefficient. This asymptotic range is such that the choice $\kappa_{j}=\mathcal{O}(1)$ or $\kappa_{j}=\infty$ in (2.1b) has a leading-order effect on the asymptotic solution to (2.1) (see section 3.1 below).

One main application of (2.1) is with regard to the determination of the mean first passage time for a diffusing particle on the unit sphere in the presence of $N$ localized partially absorbing traps. For this context, $u(x)$ in $(2.1)$ denotes the mean first passage time for an initial random walk starting from $x \in S_{\varepsilon}$, provided that we identify $M=1 / D$, where $D$ is the constant diffusivity, and set $b_{j}=0$ for $j=1, \ldots, N$ in $(2.1 \mathrm{~b})$ (cf. [35], [37]).

The second main application of (2.1) is that it represents the limiting steady-state concentration field for a three-dimensional diffusion problem posed in an asymptotically thin spherical shell, representing the thin cell membrane. Here, we imagine that a protein or signaling molecule is produced inside the cell and, subsequently, delivered by some transport mechanism to the near cell-surface region, of thickness $\delta \ll 1$, with rate $\delta M$. Biological examples of such intracellular signaling mechanisms leading to spatial gradients of a diffusible signaling molecules, such as $\mathrm{Ca}^{2+}$ or the mobile protein Rac, are discussed in the introduction of [41] and in section 3 of [30]. In [30] a numerical method for coupling surface diffusion in the cell membrane to diffusion within the cell is formulated and applied to the diffusion of Rac, which is known to exist both inside the cell and in the cell membrane. In our analysis we neglect the coupling between the cell membrane and cell interior and for definiteness consider a thin spherical shell of thickness $\delta \ll 1$ between the outer and inner surfaces at $r=1$ and $r=1-\delta$, respectively. We impose that there is no flux across $r=1$ and a prescribed flux of $\delta M$ on the inner surface $r=1-\delta$. In the thin shell we assume that there are traps $D_{\delta_{j}}$, or absorbing patches, defined in terms of spherical coordinates by

$$
D_{\delta_{j}}=\Omega_{\delta_{j}} \times[1-\delta, 1], \quad \Omega_{\delta_{j}}=\left\{(\theta, \phi) \mid\left(\theta-\theta_{j}\right)^{2}+\sin ^{2} \theta_{j}\left(\phi-\phi_{j}\right)^{2} \leq \delta^{2} a_{j}^{2}\right\},
$$

so that $\Omega_{\delta_{j}}$ is the circular projection of the trap on the surface of the sphere. The time-dependent diffusion equation in the thin spherical shell is

$$
\begin{gathered}
u_{t}=\frac{1}{r^{2}} \partial_{r}\left(r^{2} \partial_{r} u\right)+\frac{1}{r^{2} \sin ^{2} \theta} \partial_{\phi \phi} u+\frac{1}{r^{2} \sin \theta} \partial_{\theta}\left(\sin \theta \partial_{\theta} u\right), \\
1-\delta<r<1, \quad(\theta, \phi) \notin \cup_{j=1}^{N} \Omega_{\delta_{j}}, \\
u_{r}=0 \quad \text { on } r=1 ; \quad u_{r}=-\delta M \quad \text { on } r=1-\delta,
\end{gathered}
$$

with a partially absorbing boundary condition on the sides of each $D_{\delta_{j}}$. In the thin shell limit $\delta \ll 1$, we introduce $\rho=\delta^{-1}(1-r)$ and $v(\rho, \theta, \phi, t)=u(1-\delta \rho, \theta, \phi, t)$, 
and we expand $v=v_{0}+\delta v_{1}+\delta^{2} v_{2}+\cdots$. Upon substituting these new variables into (2.2) and collecting powers of $\delta$, we obtain that $v_{j}=v_{j}(\theta, \phi, t)$ for $j=0,1$, and that $v_{2}$ satisfies

$$
\begin{gathered}
\partial_{\rho \rho} v_{2}=v_{0 t}-\frac{1}{\sin ^{2} \theta} \partial_{\phi \phi} v_{0}-\frac{1}{\sin \theta} \partial_{\theta}\left(\sin \theta \partial_{\theta} v_{0}\right) ; \\
\partial_{\rho} v_{2}=0 \quad \text { on } \rho=0, \quad \partial_{\rho} v_{2}=M \quad \text { on } \rho=1 .
\end{gathered}
$$

From the solvability condition for $v_{2}$, we obtain that $v_{0}$ satisfies

$$
v_{0 t}=\frac{1}{\sin ^{2} \theta} \partial_{\phi \phi} v_{0}+\frac{1}{\sin \theta} \partial_{\theta}\left(\sin \theta \partial_{\theta} v_{0}\right)+M, \quad(\theta, \phi) \notin \cup_{j=1}^{N} \Omega_{\delta_{j}},
$$

with partially absorbing boundary conditions on $\Omega_{\delta_{j}}$. The steady-state problem for (2.3) is precisely $(2.1)$.

We may also consider the time-dependent version associated with (2.1), formulated as

$$
\begin{gathered}
w_{t}=D \triangle_{s} w+M, \quad x \in S_{\varepsilon} \equiv S \backslash \cup_{j=1}^{N} \Omega_{\varepsilon_{j}}, \\
\varepsilon \nabla_{s} w \cdot \hat{n}+\kappa_{j}\left(w-b_{j}\right)=0, \quad x \in \partial \Omega_{\varepsilon_{j}}, \\
w=w_{0} \quad \text { when } \quad t=0 .
\end{gathered}
$$

In order to study the approach to the steady-state solution $u$, we write $w=u+$ $\exp (-\sigma D t) \psi$ and obtain the following eigenvalue problem for $\sigma$, with normalized eigenfunction $\psi$ :

$$
\begin{gathered}
\triangle_{s} \psi+\sigma \psi=0, \quad x \in S_{\varepsilon} \equiv S \backslash \cup_{j=1}^{N} \Omega_{\varepsilon_{j}}, \\
\varepsilon \nabla_{s} \psi \cdot \hat{n}+\kappa_{j} \psi=0, \quad x \in \partial \Omega_{\varepsilon_{j}}, \\
\int_{S} \psi^{2} d s=1 .
\end{gathered}
$$

In section 4 we will asymptotically calculate the smallest, or principal, eigenvalue of (2.5) in the limit of small trap radii. Some bounds on the principal eigenvalue were given in $[9]$.

Numerical methods for problems related to (2.1) on the surface of the sphere were formulated in [18]. Our approach in sections 3 and 4 is to use the method of matched asymptotic expansions in the limit $\varepsilon \rightarrow 0$ of small trap radii to asymptotically solve (2.1) and (2.5), respectively. Our asymptotic analysis relies on some detailed properties of the Green's function for the Laplacian and the Helmholtz operators on the surface of the unit sphere. These key properties are summarized in Appendix A.

2.1. Previous results. In [29] (see also [2], [17], [42], [37]) the mean first passage time for a diffusing particle on a sphere of radius $L$ with one perfectly absorbing trap was considered. By centering the trap conveniently at the north pole, (2.1) with $M=L^{2} / D$ reduces to the following ODE for $u(\theta)$ :

$$
\frac{1}{\sin \theta} \frac{\partial}{\partial \theta}\left(\sin \theta \partial_{\theta} u\right)=-\frac{L^{2}}{D}, \quad \theta_{c}<\theta<\pi
$$

with $u\left(\theta_{c}\right)=0$ and $u^{\prime}(\pi)=0$. The solution to (2.6) is readily found to be (cf. [29], $[17],[37])$

$$
u=\frac{L^{2}}{D} \log \left(\frac{1-\cos \theta}{1-\cos \theta_{c}}\right) .
$$

Copyright $@$ by SIAM. Unauthorized reproduction of this article is prohibited. 
Since the area of the trap is $\left|\Omega_{\varepsilon_{1}}\right|=2 \pi\left(1-\cos \theta_{c}\right)$ we let $\theta_{c} \equiv \varepsilon \ll 1$ so that $\left|\Omega_{\varepsilon_{1}}\right| \sim \pi \varepsilon^{2}$. With $\theta_{c}=\varepsilon \ll 1,(2.7)$ becomes

$$
u \sim \frac{L^{2}}{D}[-2 \log \varepsilon+\log 2+\log (1-\cos \theta)] .
$$

The corresponding averaged mean first passage time $\bar{u}=\frac{1}{4 \pi} \int_{S} u d s$, as given in equation (5.6) of [37], is

$$
\bar{u} \sim \frac{L^{2}}{D}[-2 \log \varepsilon+2 \log 2-1] .
$$

In section 3 below these results are readily recovered as special cases of our analysis of (2.1) with $N$ traps.

In [47] (see also [7]), the principal eigenvalue of (2.5) was calculated asymptotically for the case of one perfect or partially absorbing trap. By centering the trap at the south pole as in [47], (2.5) reduces to

$$
\partial_{\theta \theta} \psi+\cot (\theta) \partial_{\theta} \psi+\sigma \psi=0, \quad 0<\theta<\theta_{c},
$$

with $\psi$ singularity-free at $\theta=0$ and with either $\psi\left(\theta_{c}\right)=0$ for a perfectly absorbing trap or $\partial_{\theta} \psi+\kappa_{1} \psi=0$ at $\theta=\theta_{c}$ for a partially absorbing trap. Here $\theta_{c} \approx \pi$. In equations (9) and (15) of [47], the following formulas for the principal eigenvalue $\sigma(\varepsilon)$ were derived when $\theta_{c} \approx \pi$ :

$$
\sigma(\varepsilon) \sim\left[\frac{2}{1-\cos \theta_{c}} \log \left(\frac{2}{1+\cos \theta_{c}}\right)-1\right]^{-1} \quad \text { (perfectly absorbing) },
$$

$\sigma(\varepsilon) \sim\left[\frac{1}{\kappa_{1}} \tan \left(\frac{\theta_{c}}{2}\right)+\frac{2}{1-\cos \theta_{c}} \log \left(\frac{2}{1+\cos \theta_{c}}\right)-1\right]^{-1} \quad$ (partially absorbing).

Upon defining $\varepsilon$ by $\theta_{c}=\pi-\varepsilon$, we have that for $\varepsilon \ll 1$, (2.10) are equivalent to

$$
\begin{gathered}
\sigma \sim \frac{\mu}{2}+\mu^{2}\left(-\frac{\log 2}{2}+\frac{1}{4}\right) ; \\
\mu=-\frac{1}{\log \varepsilon} \quad \text { (perfect), } \quad \mu=-\frac{1}{\log \left[\varepsilon e^{-1 / \kappa_{1}}\right]} \quad \text { (partial). }
\end{gathered}
$$

In section 4 below these 1-trap results are readily recovered as special cases of our analysis of (2.5) with $N$ traps.

3. Steady-state surface diffusion: Mean first passage time. We first construct the asymptotic solution to (2.1) for $N$ identical perfectly absorbing "circular" traps of a small common radius $\varepsilon a$, formulated as

$$
\triangle_{s} u=-M, \quad x \in S_{\varepsilon} \equiv S \backslash \cup_{j=1}^{N} \Omega_{\varepsilon_{j}} ; \quad u=b_{j}, \quad x \in \partial \Omega_{\varepsilon_{j}} .
$$

Here $M=M(\theta, \phi)$ is a given function, and each $\Omega_{\varepsilon_{j}}$ for $j=1, \ldots, N$ is assumed to be a small "circular" cap centered at $\left(\theta_{j}, \phi_{j}\right)$ with boundary

$$
\partial \Omega_{\varepsilon_{j}} \equiv\left\{(\theta, \phi) \mid\left(\theta-\theta_{j}\right)^{2}+\sin ^{2}\left(\theta_{j}\right)\left(\phi-\phi_{j}\right)^{2}=\varepsilon^{2} a^{2}\right\} .
$$

The area of $\Omega_{\varepsilon_{j}}$, denoted by $\left|\Omega_{\varepsilon_{j}}\right|$, is $\left|\Omega_{\varepsilon_{j}}\right|=\pi \varepsilon^{2} a^{2}$. We also assume that $M$ and $u$ are $2 \pi$ periodic in $\phi$ and have no singularities at the poles $\theta=0$ and $\theta=\pi$. Although, 
without loss of generality, we can set $a=1$ in the definition of the common trap radius $\varepsilon a$, we choose to keep the product $\varepsilon a$ in the analysis below in order to more readily compare our results with those in section 4.1 below for the more general problem involving traps of different radii.

We will construct the solution to (3.1) by the method of matched asymptotic expansions. The solution in the inner, or local, region near each trap is determined and then matched to an outer, or global, solution, valid away from $\mathcal{O}(\varepsilon)$ neighborhoods near each trap. In the inner region near the $j$ th trap we introduce the local variables

$$
\hat{\theta}=\varepsilon^{-1}\left(\theta-\theta_{j}\right), \quad \hat{\phi}=\varepsilon^{-1}\left(\phi-\phi_{j}\right), \quad v(\hat{\theta}, \hat{\phi})=u\left(\theta_{j}+\varepsilon \hat{\theta}, \phi_{j}+\varepsilon \hat{\phi}\right) .
$$

Upon substituting (3.2) into (3.1a) we obtain that

$$
\left(\partial_{\hat{\theta} \hat{\theta}}+\frac{1}{\sin ^{2} \theta_{j}} \partial_{\hat{\phi} \hat{\phi}}\right) v=\mathcal{O}(\varepsilon) \quad \text { outside } \quad \Omega_{j} ; \quad v=b_{j} \quad \text { on } \quad \partial \Omega_{j} .
$$

Here $\Omega_{j}=\varepsilon^{-1} \Omega_{\varepsilon_{j}}$ is the magnified trap region defined by $\hat{\theta}^{2}+\sin ^{2}\left(\theta_{j}\right) \hat{\phi}^{2} \leq a^{2}$. We then introduce the new variables $y_{1}$ and $y_{2}$ by

$$
y_{1}=\sin \left(\theta_{j}\right) \hat{\phi}, \quad y_{2}=\hat{\theta} .
$$

Therefore, with an $\mathcal{O}(\varepsilon)$ error, and with $y=\left(y_{1}, y_{2}\right)$, we obtain to leading order in $\varepsilon$ that $v$ satisfies

$$
v_{y_{1} y_{1}}+v_{y_{2} y_{2}}=0, \quad y \notin \Omega_{j} ; \quad v=b_{j}, \quad y \in \partial \Omega_{j},
$$

where $\partial \Omega_{j} \equiv\left\{\left(y_{1}, y_{2}\right) \mid y_{1}^{2}+y_{2}^{2}=a^{2}\right\}$. In terms of an unknown constant $A_{j}$, the solution to $(3.5)$ is

$$
v_{j}=b_{j}+A_{j} \log (|y| / a)
$$

where $|y|=\left(y_{1}^{2}+y_{2}^{2}\right)^{1 / 2}$. By rewriting (3.6) in terms of outer variables $\hat{y}=\varepsilon y$ and recalling that $\left|x-x_{j}\right|=\hat{y}+o$ (1) from (A.6), we obtain matching conditions for the outer solution as $x \rightarrow x_{j}$ for $j=1, \ldots, N$.

The constant $A_{j}$ has the interpretation as the average flux $\mathcal{F}_{j}$ of $u$ that passes across the boundary $\partial \Omega_{j}$ of the $j$ th trap. Upon using (3.6), we identify $A_{j}$ as

$$
\mathcal{F}_{j} \equiv \frac{1}{2 \pi} \int_{\partial \Omega_{j}} \partial_{\rho} v d s=A_{j}
$$

In the outer region, the $j$ th trap shrinks to the point $x_{j}$ as $\varepsilon \rightarrow 0$. With regard to the outer solution the influence of each trap is, in effect, determined by a certain singularity behavior at each $x_{j}$ that results from the asymptotic matching of the outer solution to the far-field behavior of the inner solution. In this way, we obtain that the outer solution satisfies

$$
\begin{gathered}
\triangle_{s} u=-M, \quad x \in S \backslash\left\{x_{1}, \ldots, x_{N}\right\}, \\
u \sim b_{j}+\frac{A_{j}}{\mu}+A_{j} \log \left|x-x_{j}\right| \quad \text { as } \quad x \rightarrow x_{j}, \quad j=1, \ldots, N,
\end{gathered}
$$

where $u$ is singularity-free at the poles $\theta=0, \pi$ and is $2 \pi$ periodic in $\phi$. Here $A_{j}=$ $A_{j}(\mu)$, where $\mu$ is defined by

$$
\mu \equiv-1 / \log (\varepsilon a)
$$

Copyright (C) by SIAM. Unauthorized reproduction of this article is prohibited. 
We remark that the singularity behavior in (3.8b) specifies both the regular and the singular parts of a Coulomb singularity. Consequently, each singularity behavior provides one constraint for the determination of a linear system for the source strengths $A_{j}$ for $j=1, \ldots, N$.

We decompose the solution to (3.8) in the form

$$
u=u_{p}-2 \pi \sum_{i=1}^{N} A_{i} G\left(x ; x_{i}\right)+\chi,
$$

in terms of an additional constant $\chi=\chi(\mu)$ to be determined. In (3.10), $u_{p}$ is the unique solution to

$$
\triangle_{s} u_{p}=-M+\bar{M}, \quad x \in S ; \quad \int_{S} u_{p} d s=0,
$$

where $\bar{M}$ is defined by

$$
\bar{M} \equiv \frac{1}{4 \pi} \int_{S} M d s=\frac{1}{4 \pi} \int_{0}^{2 \pi} \int_{0}^{\pi} M(\theta, \phi) \sin \theta d \theta d \phi .
$$

Moreover, in (3.10), $G\left(x ; x_{j}\right)$ is the Green's function of (A.2) given explicitly by (A.5). Since $\int_{S} u_{p} d s=\int_{S} G d s=0$, it follows that $\chi$ is the average of $u$ over the sphere, i.e.,

$$
\chi=\frac{1}{4 \pi} \int_{S} u d s .
$$

To determine a linear algebraic system for $A_{j}$, for $j=1, \ldots, N$, and for $\chi$, we first use (A.5) for $G$ and then expand (3.10) as $x \rightarrow x_{j}$ to obtain

$$
u \sim u_{p}\left(x_{j}\right)-2 \pi A_{j}\left[-\frac{1}{2 \pi} \log \left|x-x_{j}\right|+R\right]-2 \pi \sum_{\substack{i=1 \\ i \neq j}}^{N} A_{i} G_{j i}+\chi \quad \text { as } \quad x \rightarrow x_{j},
$$

where $G_{j i} \equiv G\left(x_{j} ; x_{i}\right)$ and $R \equiv-(4 \pi)^{-1}[1-2 \log 2]$ is the regular part of $G$ given in (A.5). Upon comparing this expression with the required singularity behavior in (3.8b), we obtain the following $N$ linear equations:

$$
A_{j}\left(\frac{1}{\mu}+2 \pi R\right)+2 \pi \sum_{\substack{i=1 \\ i \neq j}}^{N} G_{j i} A_{i}=u_{p}\left(x_{j}\right)-b_{j}+\chi, \quad j=1, \ldots, N .
$$

We then use the divergence theorem on (3.8) to obtain the additional equation

$$
\sum_{j=1}^{N} A_{j}=2 \bar{M}
$$

where $\bar{M}$ is the mean of $M$ defined in (3.12). The system (3.15) yields $N+1$ equations for the $N+1$ unknowns $A_{j}, j=1, \ldots, N$, and $\chi$. Upon substituting (A.5) for $G$ into (3.15), we can write this system as

$$
\begin{aligned}
A_{j}-\mu \sum_{\substack{i=1 \\
i \neq j}}^{N} A_{i} \log \left|x_{i}-x_{j}\right| & =\mu\left[u_{p}\left(x_{j}\right)-b_{j}\right]+\mu[\chi-\bar{M}(2 \log 2-1)], \quad j=1, \ldots, N ; \\
\sum_{j=1}^{N} A_{j} & =2 \bar{M} .
\end{aligned}
$$

Copyright $@$ by SIAM. Unauthorized reproduction of this article is prohibited. 
We can then decouple (3.16) and rewrite the system in matrix form. In this way, we obtain the following main result.

Principal ReSult 3.1. For the case of $N$ identical perfect "circular" traps of a common radius $\varepsilon a$, the asymptotic solution to (3.1) in the outer region $\left|x-x_{j}\right| \gg \mathcal{O}(\varepsilon)$ for $j=1, \ldots, N$ is given by (3.10), where $u_{p}(x)$ is the solution to (3.11), and where $\mathcal{A}^{t}=\left(A_{1}, \ldots, A_{N}\right)$ is the solution of the $N \times N$ linear system

$$
\left(I-\mu(I-E) \mathcal{G}_{0}\right) \mathcal{A}=\frac{2 \bar{M}}{N} e+\mu(I-E) f, \quad \mu \equiv \frac{-1}{\log (\varepsilon a)} .
$$

Here $I$ is the $N \times N$ identity matrix, and we have defined the vectors $e$ and $f$ and the matrix $E$ by

$$
e^{t} \equiv(1, \ldots, 1)^{t}, \quad f^{t} \equiv\left(u_{p}\left(x_{1}\right)-b_{1}, \ldots, u_{p}\left(x_{N}\right)-b_{N}\right), \quad E \equiv \frac{1}{N} e e^{t},
$$

where $t$ denotes transpose. In addition, $\mathcal{G}_{0}$ is the symmetric Green's function matrix with zero diagonal entries, i.e., $\mathcal{G}_{0 j j}=0$ for $j=1, \ldots, N$, and $\mathcal{G}_{0 i j}=\log \left|x_{i}-x_{j}\right|$ for $i \neq j$. In terms of $\mathcal{A}, \chi$ in (3.10) is given explicitly by

$$
\chi=\frac{2 \bar{M}}{\mu N}+(2 \log 2-1) \bar{M}-\frac{1}{N} e^{t} \mathcal{G}_{0} \mathcal{A}-\frac{1}{N} e^{t} f .
$$

We remark that our asymptotic solution contains all logarithmic terms in powers of $\mu$ in the asymptotic solution of (3.1). As such, (3.17) has in effect "summed" all of the logarithmic terms in the expansion of the solution.

For the special case where $b_{j}=0$ for $j=1, \ldots, N$ and where $M$ is constant, so that $u_{p} \equiv 0$ from (3.11), the system (3.15) can be simplified considerably. As remarked in section 2, this special case with $M=1 / D$ is directly relevant to calculating the mean first passage time on a unit sphere with identical "circular" traps centered at locations $x_{1}, \ldots, x_{N}$ on the sphere. Upon retaining only the first few terms in powers of $\mu$ from (3.17), we obtain that Principal Result 3.1 can be reduced to the following rather explicit main result.

Corollary 3.1. Under the conditions of Principal Result 3.1, let $M$ be a constant and let $b_{j}=0$ for $j=1, \ldots, N$. Then, the asymptotic solution to (3.1) in the outer region $\left|x-x_{j}\right| \gg \mathcal{O}(\varepsilon)$ for $j=1, \ldots, N$ is given by

$$
u(x)=-2 \pi \sum_{j=1}^{N} A_{j} G\left(x ; x_{j}\right)+\chi .
$$

For $\mu \ll 1, A_{j}$ is the $j$ th component of $\mathcal{A}$, which is given asymptotically by

$$
\mathcal{A}=\frac{2 M}{N}\left[e+\mu(I-E) \mathcal{G}_{0} e+\mu^{2}\left[(I-E) \mathcal{G}_{0}\right]^{2} e+\mathcal{O}\left(\mu^{3}\right)\right] .
$$

In addition, the average of $u$, given by $\bar{u}=\chi$, satisfies

$\bar{u}=\chi=\frac{2 M}{N \mu}+M\left[(2 \log 2-1)-\frac{4}{N^{2}} p\left(x_{1}, \ldots, x_{N}\right)\right]-\frac{2 \mu M}{N^{2}} e^{t} \mathcal{G}_{0}(I-E) \mathcal{G}_{0} e+\mathcal{O}\left(\mu^{2}\right)$,

where the discrete energy $p\left(x_{1}, \ldots, x_{N}\right)$ is defined by

$$
p\left(x_{1}, \ldots, x_{N}\right) \equiv \sum_{i=1}^{N} \sum_{j>i}^{N} \log \left|x_{i}-x_{j}\right| .
$$

Copyright $@$ by SIAM. Unauthorized reproduction of this article is prohibited. 
We remark that it is readily verified that our system (3.15) reproduces the mean first passage time result (2.8) of [29] for the case of one trap centered, conveniently, at the north pole $x_{1}$ of the unit sphere. To see this, we set $N=1, b_{1}=0$, and $M=1 / D$ in (3.15) to obtain that $A_{1}=2$ and $\chi=4 \pi R+2 / \mu$. Therefore, upon using $\left|x-x_{1}\right|^{2}=2-2 \cos \theta$ together with (A.5) for $G$, we obtain that (3.18a) becomes

$$
u \sim-4 \pi G\left(x ; x_{1}\right)+\frac{2}{\mu}+4 \pi R=2 \log \left|x-x_{j}\right|+\frac{2}{\mu}=\log (1-\cos \theta)+\log 2+\frac{2}{\mu},
$$

which agrees with (2.8). For this special case, $\bar{u}$ from (3.18c) becomes

$$
\bar{u}=\chi=-2 \log (\varepsilon a)+2 \log 2-1,
$$

which agrees with equation (5.6) of [37] written in (2.9).

In the context of the mean first passage time problem, the constant $\chi$ in $(3.18 \mathrm{a})$ and $(3.18 \mathrm{c})$ has the immediate interpretation as the mean first passage time averaged with respect to an initial uniform distribution of starting points on the unit sphere. Upon setting $M=1 / D$, where $D$ is the diffusion coefficient on the unit sphere, the first two terms for the averaged mean first passage time are written using (3.18c) as

$$
\begin{aligned}
\bar{u} & =\frac{1}{D}\left[\frac{2}{N \mu}+(2 \log 2-1)-\frac{4}{N^{2}} p\left(x_{1}, \ldots, x_{N}\right)+\mathcal{O}\left(\frac{\mu}{N^{2}}\right)\right], \\
p\left(x_{1}, \ldots, x_{N}\right) & \equiv \sum_{i=1}^{N} \sum_{j>i}^{N} \log \left|x_{i}-x_{j}\right| .
\end{aligned}
$$

Consequently, the problem of determining the spatial configuration $\left\{x_{1}, \ldots, x_{N}\right\}$ of trap locations that minimizes the average mean first passage time is equivalent in the limit $\mu \ll 1$ to the well-known discrete optimization problem, popularized by Whyte in 1952 [48], of finding the set of points $\left\{x_{1}, \ldots, x_{N}\right\}$ that maximize the discrete energy $p\left(x_{1}, \ldots, x_{N}\right)$. These points are referred to as either logarithmic points [11] or elliptic Fekete points [39], with mathematical origins in [16]. For small values of $N$, such points can be found by detailed geometrical constructions (see [11] for $N=5$ and [28] for $N=6$ ). In particular, for $N=5$, it was proved in [11] that the optimal configuration for $p\left(x_{1}, \ldots, x_{5}\right)$ consists of points at the north and south poles, together with three points forming an equilateral triangle on the equator. In [3] and [39] different numerical methods are used to compute the optimal energy $\max p\left(x_{1}, \ldots, x_{N}\right)$ for a range of values of $N$. As $N$ increases, it becomes increasingly difficult to numerically compute the globally optimizing configuration owing to the very large number of local maxima. However, for $N \rightarrow \infty$, equation (4.5) of [32] (see also [3] and [31]) gives the asymptotics of the optimal energy as

$$
\begin{gathered}
\max p\left(x_{1}, \ldots, x_{N}\right) \sim \frac{1}{4} \log \left(\frac{4}{e}\right) N^{2}+\frac{1}{4} N \log N+l_{1} N+l_{2}, \\
N \rightarrow \infty, \quad l_{1}=0.02642, \quad l_{2}=0.1382 .
\end{gathered}
$$

The first two terms in (3.22) are rigorous results, while the last two terms are fitted to results obtained from a numerical optimization of $p\left(x_{1}, \ldots, x_{N}\right)$ (cf. [31], [32], [3]). Upon substituting (3.22) into (3.21), we obtain that the minimum of the averaged mean first passage time in the limit of large $N$ has the scaling law

$$
\min \bar{u} \sim \frac{1}{D}\left[\frac{2}{N \mu}-\left(\frac{\log N}{N}+\frac{4 l_{1}}{N}+\mathcal{O}\left(N^{-2}\right)\right)\right], \quad l_{1}=0.026422 .
$$


For $N \gg 1$, this result is asymptotically valid only when $\varepsilon$ is sufficiently small so that the correction term given in the round brackets in (3.23) is smaller than the first term. This requires that $\log N \ll-2 \log (\varepsilon a)$, which reduces to the assumption $N \varepsilon^{2} a^{2} \ll 1$, i.e., that the total area occupied by the $N$ traps is asymptotically small compared to the $\mathcal{O}(1)$ surface area of the sphere. We can then readily rewrite (3.23) to obtain the simple explicit result that for $N \gg 1$, but in the small area fraction limit $N \varepsilon^{2} a^{2} \ll 1$, $\min \bar{u}$ has the asymptotic behavior

$$
\min \bar{u} \sim \frac{1}{N D}\left[-\log \left(\frac{\sum_{j=1}^{N}\left|\Omega_{\varepsilon_{j}}\right|}{|S|}\right)-4 l_{1}-\log 4+\mathcal{O}\left(N^{-1}\right)\right], \quad l_{1}=0.026422 .
$$

Here $\sum_{j=1}^{N}\left|\Omega_{\varepsilon_{j}}\right|=N \pi \varepsilon^{2} a^{2}$ is the total trap area, while $|S|=4 \pi$ is the surface area of the unit sphere $S$. The result (3.24) can then be used to define an effective diffusion coefficient.

3.1. Partially absorbing traps. Next, we consider the case of partially absorbing traps of different radii. The boundary condition on the $j$ th trap in (3.1a) is replaced by

$\varepsilon \nabla_{s} u \cdot \hat{n}+\kappa_{j}\left(u-b_{j}\right)=0, \quad x \in \partial \Omega_{\varepsilon_{j}} \equiv\left\{(\theta, \phi) \mid\left(\theta-\theta_{j}\right)^{2}+\sin ^{2}\left(\theta_{j}\right)\left(\phi-\phi_{j}\right)^{2}=\varepsilon^{2} a_{j}^{2}\right\}$.

For this nonidentical multitrap case, we can uniquely define $\varepsilon$ as the average dimensionless radius of the collection of traps, so that $N^{-1} \sum_{j=1}^{N} a_{j}=1$.

In place of (3.5), for this more general problem we obtain the inner problem

$$
v_{y_{1} y_{1}}+v_{y_{2} y_{2}}=0, \quad y \notin \Omega_{j} ; \quad-v_{\rho}+\kappa_{j}\left(v-b_{j}\right)=0, \quad y \in \partial \Omega_{j},
$$

where $\Omega_{j} \equiv \varepsilon^{-1} \Omega_{\varepsilon_{j}}, y=\left(y_{1}, y_{2}\right)$, with $\rho=|y|, y_{1}=\varepsilon^{-1}\left(\phi-\phi_{j}\right) \sin \theta_{j}$, and $y_{2}=$ $\varepsilon^{-1}\left(\theta-\theta_{j}\right)$. The solution to $(3.26)$ in terms of some unknown constant $A_{j}$ is

$$
v=b_{j}+A_{j} \log \left(\rho / \beta_{j}\right), \quad \mu_{j} \equiv-\frac{1}{\log \left(\varepsilon \beta_{j}\right)}, \quad \beta_{j} \equiv a_{j} \exp \left(-1 / a_{j} \kappa_{j}\right) .
$$

Upon rewriting (3.27) in outer variables, we obtain that the corresponding outer solution satisfies

$$
\begin{gathered}
\triangle_{s} u=-M, \quad x \in S \backslash\left\{x_{1}, \ldots, x_{N}\right\}, \\
u \sim b_{j}+\frac{A_{j}}{\mu_{j}}+A_{j} \log \left|x-x_{j}\right| \quad \text { as } \quad x \rightarrow x_{j}, \quad j=1, \ldots, N,
\end{gathered}
$$

where $u$ is singularity-free at the poles $\theta=0, \pi$ and is $2 \pi$ periodic in $\phi$. The solution to (3.28) is decomposed as in (3.10), where in place of (3.16) we obtain that $A_{j}$, for $j=1, \ldots, N$, and $\chi$ in (3.10) now satisfy the linear system

$$
\begin{aligned}
\frac{A_{j}}{\mu_{j}}-\sum_{\substack{i=1 \\
i \neq j}}^{N} A_{i} \log \left|x_{i}-x_{j}\right| & =u_{p}\left(x_{j}\right)-b_{j}+\chi-\bar{M}(2 \log 2-1), \quad j=1, \ldots, N ; \\
\sum_{j=1}^{N} A_{j} & =2 \bar{M} .
\end{aligned}
$$

Copyright (c) by SIAM. Unauthorized reproduction of this article is prohibited. 
In particular, $\chi$ is now given by

$$
\chi=\frac{2 \bar{M}}{N \bar{\mu}}+(2 \log 2-1) \bar{M}-\frac{1}{N \bar{\mu}} e^{t} \mathcal{U} \mathcal{G}_{0} \mathcal{A}-\frac{1}{N \bar{\mu}} e^{t} \mathcal{U} f
$$

where $\mathcal{U}=\operatorname{diag}\left(\mu_{1}, \ldots, \mu_{N}\right), N \bar{\mu}=\operatorname{Trace}(\mathcal{U})$, and $\mathcal{A}$ is determined from

$$
\left(I-\left(I-\frac{1}{\bar{\mu}} \mathcal{U} E\right) \mathcal{U} \mathcal{G}_{0}\right) \mathcal{A}=\frac{2 \bar{M}}{N \bar{\mu}} \mathcal{U} e+\left(I-\frac{1}{\bar{\mu}} \mathcal{U} E\right) \mathcal{U} f .
$$

For the special case where $M$ is a constant and $b_{j}=0$ for $j=1, \ldots, N$, i.e., $f \equiv 0$, we can readily obtain simple asymptotic formulae by solving (3.30) asymptotically for $\mu_{j} \ll 1$. The result is as follows.

Principal Result 3.2. Consider $N$ partially absorbing traps of radii $\varepsilon a_{j} \ll 1$ centered at $x_{j}$, for $j=1, \ldots, N$, on the unit sphere. Then, the asymptotic solution to (3.1a) with boundary conditions (3.25) is given in the outer region $\left|x-x_{j}\right| \gg \mathcal{O}(\varepsilon)$ for $j=1, \ldots, N$ by (3.18a), where $A_{j}$ for each $j=1, \ldots, N$ in (3.18a) is given asymptotically by

$$
A_{j}=\frac{2 M \mu_{j}}{N \bar{\mu}}\left[1+\sum_{\substack{i=1 \\ i \neq j}}^{N} \mu_{i} \log \left|x_{i}-x_{j}\right|-\frac{2}{N \bar{\mu}} p_{w}\left(x_{1}, \ldots, x_{N}\right)+\mathcal{O}\left(|\mu|^{2}\right)\right] .
$$

In addition, $\bar{u}=\chi$ is given asymptotically by

$$
\bar{u}=\chi=\frac{2 M}{N \bar{\mu}}+M\left[(2 \log 2-1)-\frac{4}{N^{2} \bar{\mu}^{2}} p_{w}\left(x_{1}, \ldots, x_{N}\right)\right]+\mathcal{O}(|\mu|) .
$$

Here $\mu_{j}, \bar{\mu}$, and the weighted discrete energy $p_{w}\left(x_{1}, \ldots, x_{N}\right)$ are defined by

$$
\begin{gathered}
\mu_{j} \equiv-\frac{1}{\log \left(\varepsilon \beta_{j}\right)}, \quad \beta_{j} \equiv a_{j} \exp \left(-1 / a_{j} \kappa_{j}\right) ; \quad \bar{\mu} \equiv \frac{1}{N} \sum_{j=1}^{N} \mu_{j} ; \\
p_{w}\left(x_{1}, \ldots, x_{N}\right) \equiv \sum_{i=1}^{N} \sum_{j>i}^{N} \mu_{i} \mu_{j} \log \left|x_{i}-x_{j}\right| .
\end{gathered}
$$

In (3.31a) and (3.31b), $|\mu|^{2}$ and $|\mu|$ denote terms that are quadratic or linear in the $\mu_{j}$, respectively.

From (3.31b), we note that the average mean first passage time is minimized for a configuration of traps that maximize the weighted discrete energy $p_{w}\left(x_{1}, \ldots, x_{N}\right)$ on the unit sphere $\left|x_{j}\right|=1$. This discrete optimization problem has not, to our knowledge, been considered previously. We conclude this section with two remarks.

Remark 3.1. A minor modification of the analysis above is needed to treat the case of $N$ noncircular, partially absorbing traps on the boundary of a sphere. For this general case, the following inner problem replaces (3.26):

$$
v_{y_{1} y_{1}}+v_{y_{2} y_{2}}=0, \quad y \notin \Omega_{j} ; \quad-\nabla v \cdot \hat{n}+\kappa_{j}\left(v-b_{j}\right)=0, \quad y \in \partial \Omega_{j} .
$$

Here $\Omega_{j} \equiv \varepsilon^{-1} \Omega_{\varepsilon_{j}}, y=\left(y_{1}, y_{2}\right), \nabla v=\left(v_{y_{1}}, v_{y_{2}}\right)$, and $\hat{n}$ is the unit normal to $\partial \Omega_{j}$ pointing exterior to $\Omega_{j}$. For an arbitrary noncircular trap $\Omega_{j}$, (3.32) cannot be solved analytically, but has the far-field behavior (cf. [46])

$$
v \sim b_{j}+A_{j} \log \left(\rho / d_{j}\right)+\mathcal{O}(1) \quad \text { as } \quad|y|=\rho \rightarrow \infty,
$$

Copyright $@$ by SIAM. Unauthorized reproduction of this article is prohibited. 
where $d_{j}=d_{j}\left(\kappa_{j}\right)$ is some constant to be computed from the numerical solution to (3.32). For a perfectly absorbing trap, where $\kappa_{j}=\infty, d_{j}(\infty)$ is called the logarithmic capacitance of $\Omega_{j}$ (cf. [46], [33]), and explicit formulae for it are available for different trap shapes such as ellipses, squares, and equilateral triangles (cf. [33]). Numerical methods for computing $d_{j}(\infty)$ are given in [10] and [34]. It is well known that of all convex planar regions with a fixed area, the circular disk has the smallest logarithmic capacitance. The asymptotic expansion in Principal Result 3.2 still holds, provided that we replace $\mu_{j}$ there by $\mu_{j} \equiv-1 / \log \left[\varepsilon d_{j}\left(\kappa_{j}\right)\right]$.

Remark 3.2. The analysis leading to Principal Result 3.2 has assumed that the traps are well separated in the sense that $\left|x_{i}-x_{j}\right|=\mathcal{O}(1)$ for $i \neq j$. Next, we briefly consider the case where there are $M_{j}$ nonoverlapping traps clustered in an $\mathcal{O}(\varepsilon)$ ball near each $x_{j}$, for $j=1, \ldots, N$, where $N$ now denotes the number of clusters and $M_{1}+\cdots+M_{N}=n$ is the total number of traps. Then, we need only replace $\mu_{j}$ in Principal Result 3.2 with $-1 / \log \left(\varepsilon d_{j}\right)$, where, for each $j=1, \ldots, N, d_{j}$ is to be determined from the far-field behavior of the following inner problem with $M_{j}$ nonoverlapping traps:

$$
\begin{aligned}
& v_{y_{1} y_{1}}+v_{y_{2} y_{2}}=0, \quad y \notin \Omega_{j_{k}} ; \quad-\nabla v \cdot \hat{n}+\kappa_{j_{k}} v=0, \quad y \in \partial \Omega_{j_{k}}, \quad k=1, \ldots, M_{j}, \\
& (3.34 \mathrm{~b}) \quad v \sim \log |y|-\log d_{j}+o(1) \quad \text { as } \quad|y|=\left(y_{1}^{2}+y_{2}^{2}\right)^{1 / 2} \rightarrow \infty .
\end{aligned}
$$

In general, $d_{j}$ must be computed numerically from a boundary integral method. Alternatively, the analytical approach in [14] based on coupled integral equations could, in principle, be suitably modified to formulate an analytical iteration scheme to numerically determine $d_{j}$. A systematic and comprehensive theory for solving rather general classes of mixed boundary-value problems in potential theory is developed and surveyed in [15]. However, as shown in Appendix B, for the special case of two perfectly absorbing circular traps of a common radius $\varepsilon a_{j},(3.34)$ can be solved analytically by introducing bipolar coordinates. From (B.5), we obtain that $d_{j}$ can be written as the infinite sum

$$
\log d_{j}=\log \left(2 \beta_{j}\right)-\frac{\xi_{j}}{2}+\sum_{m=1}^{\infty} \frac{e^{-m \xi_{j}}}{m \cosh \left(m \xi_{j}\right)}, \quad \beta_{j} \equiv \sqrt{l_{j}^{2}-a_{j}^{2}} .
$$

Here $2 l_{j}$, with $l_{j}>a_{j}$, is the distance between the centers of the two circular traps measured in the inner $y$ variable, and $\xi_{j}$ is the unique root of $\cosh \left(\xi_{j}\right)=l_{j} / a_{j}$. A plot of $d_{j}$ versus $l_{j} / a_{j}>1$ is shown in Figure 2 for the case where $a_{j}=1 / \sqrt{2}$, for which the combined area of the two traps is $\pi \varepsilon^{2}$. Recall that for a single circle of area $\pi \varepsilon^{2}$ the logarithmic capacitance is $d=1$. From Figure 2 we obtain that $d_{j}>1$ for any $l_{j} / a_{j}$. Therefore, this plot shows that for trap areas of the same total size, the logarithmic capacitance of the two-circular-disk cluster is always larger than that of a single circular disk. Hence, the averaged mean first passage time of a two-circular-disk cluster is always smaller than that of a single circular disk of the same total area.

Finally, for the case of exactly two traps on the unit disk we show that (3.35) allows for a uniform transition between the case of two clustered traps and two wellseparated traps. For the case of two clustered traps, (3.20) with $\log (\varepsilon a)$ replaced by $\log \left(\varepsilon d_{1}\right)$ becomes

$$
\bar{u} \sim-2 \log \left(\varepsilon d_{1}\right)+2 \log 2-1,
$$




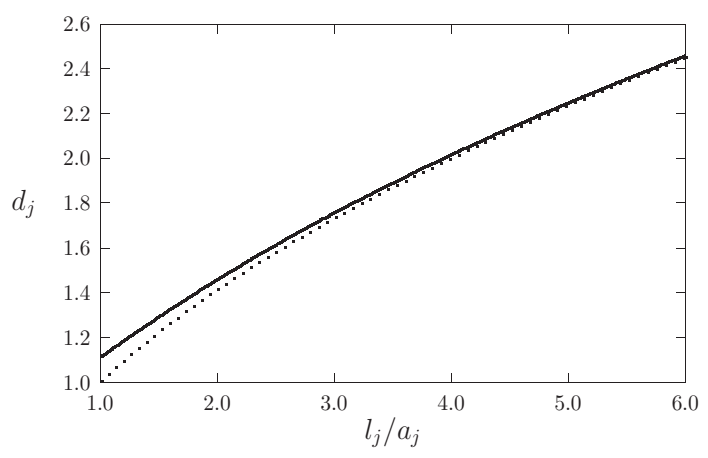

FIG. 2. Plot of the logarithmic capacitance $d_{j}$ versus $l_{j} / a_{j}$ for the two-disk cluster when $a_{j}=$ $1 / \sqrt{2}$. For this value of $a_{j}$ the total trap area for the two-disk cluster is $\pi \varepsilon^{2}$. For a single disk with area $\pi \varepsilon^{2}$ the logarithmic capacitance $d_{j 0}$ is $d_{j 0}=1$. The plot shows that $d_{j}>d_{j 0}=1$ and that $d_{j}$ is an increasing function of $l_{j} / a_{j}$. The heavy solid curve is (3.35), while the dashed curve is the asymptotic result $d_{j} \sim \sqrt{l_{j} / a_{j}}$ valid for $a_{j}=1 / \sqrt{2}$ when $l_{j} / a_{j} \gg 1$.

where $d_{1}$ is given in (3.35). In contrast, for two well-separated traps of a common radius $\varepsilon a,(3.21)$ yields

$$
\bar{u} \sim-\log (\varepsilon a)+2 \log 2-1-\log \left|x_{2}-x_{1}\right| .
$$

By taking the limit $l / a \gg 1$ in (3.35), we readily obtain from (3.35) that $d_{1} \sim$ $\log (\sqrt{2 l a})$, where $2 l=\left|x_{2}-x_{1}\right| / \varepsilon \gg 1$. With this limiting result for $d_{1}$, we obtain that (3.36) reduces to the result (3.37) for two well-separated traps.

4. The asymptotics of the principal eigenvalue. In this section we derive asymptotic expansions for the principal eigenvalue of (2.5) in the limit of small trap radii.

We first consider the special case of $N$ identical perfectly absorbing traps of a common radius $\varepsilon a$. Then, $(2.5 \mathrm{~b})$ becomes $\psi=0$ on $\partial \Omega_{\varepsilon_{j}}$ for $j=1, \ldots, N$. We let $\sigma(\varepsilon)$ denote the principal eigenvalue, and we expand $\sigma(\varepsilon)$ as

$$
\sigma(\varepsilon)=\mu \sigma_{0}+\mu^{2} \sigma_{1}+\cdots, \quad \mu \equiv-\frac{1}{\log (\varepsilon a)} .
$$

The logarithmic nature of the expansion is similar to other problems in two space dimensions with localized perturbations (cf. [5], [27], [40], and [46]). Our goal is to derive an explicit two-term expansion for $\sigma(\varepsilon)$ by deriving simple formulae for the coefficients $\sigma_{0}$ and $\sigma_{1}$.

In the outer region, away from $\mathcal{O}(\varepsilon)$ neighborhoods of the traps, we expand the outer solution for $\psi$ as

$$
\psi=\psi_{0}+\mu \psi_{1}+\mu^{2} \psi_{2}+\cdots .
$$

Upon substituting (4.1) and (4.2) into (2.5a) and (2.5c), we obtain that $\psi_{0} \equiv(4 \pi)^{-1 / 2}$, and that $\psi_{1}$ and $\psi_{2}$ satisfy

$$
\begin{aligned}
& \triangle_{s} \psi_{1}=-\sigma_{0} \psi_{0}, \quad x \in S \backslash\left\{x_{1}, \ldots, x_{N}\right\} ; \quad \int_{S} \psi_{1} d s=0 \\
& \triangle_{s} \psi_{2}=-\sigma_{1} \psi_{0}-\sigma_{0} \psi_{1}, \quad x \in S \backslash\left\{x_{1}, \ldots, x_{N}\right\} ; \quad \int_{S}\left(\psi_{1}^{2}+2 \psi_{0} \psi_{2}\right) d s=0 .
\end{aligned}
$$

Copyright $@$ by SIAM. Unauthorized reproduction of this article is prohibited. 
The singularity conditions for $\psi_{1}$ and $\psi_{2}$ as $x \rightarrow x_{j}$ are derived by matching the outer solution to appropriate inner solutions near each trap. Near the $j$ th trap we introduce inner variables by

$$
\hat{\theta} \equiv \varepsilon^{-1}\left(\theta-\theta_{j}\right), \quad \hat{\phi} \equiv \varepsilon^{-1}\left(\phi-\phi_{j}\right), \quad y_{1} \equiv \hat{\phi} \sin \theta_{j}, \quad y_{2} \equiv \hat{\theta} .
$$

Then, upon neglecting $\mathcal{O}(\varepsilon)$ terms, we obtain that $(2.5 \mathrm{a})$ and $(2.5 \mathrm{~b})$ with $\kappa_{j}=\infty$ reduces to the following inner problem for $\Psi\left(y_{1}, y_{2}\right)$ :

$$
\Psi_{y_{1} y_{1}}+\Psi_{y_{2} y_{2}}=0, \quad y \notin \Omega_{j} ; \quad \Psi=0, \quad y \in \partial \Omega_{j},
$$

where $\Omega_{j}=\left\{\left(y_{1}, y_{2}\right) \mid y_{1}^{2}+y_{2}^{2} \leq a^{2}\right\}$. The solution to (4.5) is written in terms of some unknown constant $A_{j}(\mu)$ as

$$
\Psi=\mu A_{j}(\mu) \log (|y| / a), \quad A_{j}(\mu)=A_{1 j}+\mu A_{2 j}+\mu^{2} A_{3 j}+\cdots .
$$

Here $A_{j}$ for $j=1, \ldots, 3$ are unknown coefficients that are independent of $\mu$. Upon using $|y| \sim \varepsilon^{-1}\left|x-x_{j}\right|$ to rewrite (4.6) in outer variables, we obtain the following matching condition for the outer solution for $\psi$ as $x \rightarrow x_{j}$ :

(4.7)

$\psi_{0}+\mu \psi_{1}+\mu^{2} \psi_{2} \sim A_{1 j}+\mu\left[A_{1 j} \log \left|x-x_{j}\right|+A_{2 j}\right]+\mu^{2}\left[A_{2 j} \log \left|x-x_{j}\right|+A_{3 j}\right]+\cdots$.

Therefore, (4.7) implies that $A_{1 j}=\psi_{0}=(4 \pi)^{-1 / 2}$. In addition, we obtain that $\psi_{1}$ and $\psi_{2}$ have the following singularity behavior as $x \rightarrow x_{j}$ for each $j=1, \ldots, N$ :

$$
\psi_{1} \sim \psi_{0} \log \left|x-x_{j}\right|+A_{2 j} ; \quad \psi_{2} \sim A_{2 j} \log \left|x-x_{j}\right|+A_{3 j} .
$$

Therefore, from (4.3a) and (4.8), we can write the problem for $\psi_{1}$ in terms of singular forces as

$$
\triangle_{s} \psi_{1}=-\sigma_{0} \psi_{0}+2 \pi \psi_{0} \sum_{j=1}^{N} \frac{1}{\sin \theta_{j}} \delta\left(\theta-\theta_{j}\right) \delta\left(\phi-\phi_{j}\right), \quad x \in S .
$$

Since $\left(\triangle_{s} \psi_{1}, 1\right)=0$, we obtain by the divergence theorem that $\sigma_{0}$ satisfies $-\sigma_{0} \int_{S} \psi_{0} d s$ $=-2 \pi \psi_{0} N$. This yields

$$
\sigma_{0}=\frac{N}{2} .
$$

The unique solution to (4.9) with $\int_{S} \psi_{1} d s=0$ is then written in terms of the Green's function of (A.2) as

$$
\psi_{1}=-2 \pi \psi_{0} \sum_{i=1}^{N} G\left(x ; x_{i}\right), \quad \psi_{0} \equiv(4 \pi)^{-1 / 2} .
$$

Then, by using (A.5) for $G$, we expand (4.11) as $x \rightarrow x_{j}$ to obtain that

$$
\psi_{1} \sim \psi_{0} \log \left|x-x_{j}\right|-2 \pi \psi_{0}\left[R+\sum_{i \neq j}^{N} G_{j i}\right] \quad \text { as } \quad x \rightarrow x_{j} .
$$

Here $G_{j i} \equiv G\left(x_{j} ; x_{i}\right)$ and $R$ is the regular part of $G$ given in (A.5). Upon comparing (4.12) with the required singular behavior (4.8) for $\psi_{1}$, we identify the constant $A_{2 j}$ in $(4.8)$ as

$$
A_{2 j} \equiv-2 \pi \psi_{0}\left[R+\sum_{i \neq j}^{N} G_{j i}\right]
$$

Copyright $@$ by SIAM. Unauthorized reproduction of this article is prohibited. 
Then, the problem for $\psi_{2}$ consists of (4.3b) with the singular behavior (4.8). This problem can be written in terms of singular forces as

$$
\triangle_{s} \psi_{2}=-\sigma_{1} \psi_{0}-\sigma_{0} \psi_{1}+2 \pi \sum_{j=1}^{N} \frac{A_{2 j}}{\sin \theta_{j}} \delta\left(\theta-\theta_{j}\right) \delta\left(\phi-\phi_{j}\right), \quad x \in S .
$$

Since $\left(\triangle \psi_{2}, 1\right)=\left(\psi_{1}, 1\right)=0$, we obtain from the divergence theorem and (4.13) that

$$
-\sigma_{1} \int_{S} \psi_{0} d s=-2 \pi \sum_{j=1}^{N} A_{2 j}=4 \pi^{2} \psi_{0} \sum_{j=1}^{N}\left(R+\sum_{i \neq j}^{N} G_{j i}\right) .
$$

Upon using (A.5) for $G$, we readily obtain that this expression for $\sigma_{1}$ reduces to

$$
\sigma_{1}=-\pi \sum_{j=1}^{N}\left(R+\sum_{i \neq j}^{N} G_{j i}\right)=-\pi N^{2} R+\sum_{j=1}^{N} \sum_{i>j}^{N} \log \left|x_{i}-x_{j}\right|,
$$

where $R=(4 \pi)^{-1}[2 \log 2-1]$ as given in (A.5). This determines $\sigma_{1}$ explicitly. We summarize our two-term asymptotic result for the principal eigenvalue of (2.5) for identical perfectly absorbing traps in the following statement.

Principal Result 4.1. Consider (2.5) for $N$ identical perfectly absorbing $\left(\kappa_{j}=\right.$ $\infty)$ traps of a common radius $\varepsilon$ a centered at $x_{j}$, for $j=1, \ldots, N$, on the unit sphere. Then, the principal eigenvalue $\sigma(\varepsilon)$ of $(2.5)$ is given for $\varepsilon \ll 1$ by

$$
\sigma(\varepsilon) \sim \frac{\mu N}{2}+\mu^{2}\left[-\frac{N^{2}}{4}(2 \log 2-1)+p\left(x_{1}, \ldots, x_{N}\right)\right], \quad \mu \equiv-\frac{1}{\log (\varepsilon a)},
$$

where $p\left(x_{1}, \ldots, x_{N}\right)$ is the discrete energy as given in (3.18d).

As a remark, for the special case of one perfectly absorbing trap where $N=1$, (4.17) reduces to

$$
\sigma(\varepsilon) \sim \frac{\mu}{2}+\frac{\mu^{2}}{4}(1-2 \log 2) .
$$

This result agrees with the result (2.11) of section 2, as originally derived in [47].

There is a simple relationship between (4.17) for the principal eigenvalue $\sigma(\varepsilon)$ and the averaged mean first passage time $\bar{u}=\chi$ in (3.18c). Upon setting $M=1$ in (3.18c) for simplicity, we claim that

$$
\bar{u}=\chi=\frac{1}{\sigma}+\mathcal{O}(\mu) .
$$

This relationship is readily verified by comparing (3.18c) and (4.17). To derive (4.19), we expand the solution to (2.1) with $M=1$ in terms of all of the normalized eigenfunctions of (2.5). In the usual way, we obtain an eigenfunction expansion representation for $u$, and from it calculate $\bar{u}$ as

$$
\bar{u}=\chi=\frac{1}{4 \pi}\left[\frac{\left(\psi_{1}, 1\right)^{2}}{\sigma_{1}}+\sum_{j=2}^{\infty} \frac{\left(\psi_{j}, 1\right)^{2}}{\sigma_{j}}\right] .
$$

Here $(u, v) \equiv \int_{S} u v d s$, and we have labeled $\sigma_{j}(\varepsilon)$ and $\psi_{j}(x ; \varepsilon)$ as the $j$ th normalized eigenpair of (2.5). Therefore, in the limit of small trap radii $\varepsilon \rightarrow 0$, the higher 
modes with $j \geq 2$ contribute $\left(\psi_{j}, 1\right)=\mathcal{O}(\mu)$ and $\sigma_{j}=\mathcal{O}(1)$ to the sum in (4.20). This estimate for $\left(\psi_{j}, 1\right)$ when $j \geq 2$ follows from the orthogonality of eigenfunctions and the fact that 1 is an $\mathcal{O}(\mu)$ close approximation to the principal (unnormalized) eigenfunction $\psi_{1}$ when $\varepsilon \ll 1$. However, for the $j=1$ mode, we obtain from the outer expansion (4.2) that $\left(\psi_{1}, 1\right) \sim \sqrt{4 \pi}+\mu^{2}\left(\psi_{12}, 1\right)$ and $\sigma_{1}=\mathcal{O}(\mu)$ from (4.17). Here, with a minor change in notation, $\psi_{12}$ is the solution to (4.14). With these estimates, (4.20) readily yields (4.19).

Finally, as in section 3, we note that the principal eigenvalue $\sigma(\varepsilon)$ is maximized at the elliptic Fekete points $\left\{x_{1}, \ldots, x_{N}\right\}$ that maximize the discrete energy $p\left(x_{1}, \ldots, x_{N}\right)$. When $\left\{x_{1}, \ldots, x_{N}\right\}$ is chosen to maximize $p\left(x_{1}, \ldots, x_{N}\right)$, we obtain from the optimal energy (3.22) that (4.17) for $N \gg 1$ becomes

$$
\max \sigma(\varepsilon) \sim \frac{\mu N}{2}+\mu^{2}\left[\frac{N}{4} \log N+l_{1} N+l_{2}\right]
$$

where $l_{1}$ and $l_{2}$ were given in (3.22). This scaling law is valid only when $\mu N \ll 1$, so that $\sigma \ll 1$.

4.1. Summing the logarithmic series for the principal eigenvalue. Principal Result 4.1 gives a two-term expansion in powers of $\mu$ for $\sigma(\varepsilon)$ for the case of identical perfectly absorbing traps. We now formulate a hybrid asymptotic-numerical matrix eigenvalue problem that has the effect of summing all of the logarithmic terms in the asymptotic expansion of $\sigma(\varepsilon)$. In doing so we will obtain $\sigma(\varepsilon)$ accurate up to insignificant algebraically small terms in $\varepsilon$. In our analysis, we will consider the generalized problem of (2.5) with $N$ partially absorbing circular traps of different radii. As a by-product of our analysis, we will derive an explicit two-term expansion for $\sigma(\varepsilon)$, similar to that in Principal Result 4.1, for the case of partially absorbing traps of different radii.

In the inner region near the $j$ th trap for (2.5), we obtain the following inner problem in place of (4.5):

$$
\Psi_{y_{1} y_{1}}+\Psi_{y_{2} y_{2}}=0, \quad y \notin \Omega_{j} ; \quad-\Psi_{\rho}+\kappa_{j} \Psi=0, \quad y \in \partial \Omega_{j} .
$$

Here $\Omega_{j}=\left\{\left(y_{1}, y_{2}\right) \mid y_{1}^{2}+y_{2}^{2} \leq a_{j}^{2}\right\}, \rho \equiv\left(y_{1}^{2}+y_{2}^{2}\right)^{1 / 2}$, where $y_{1}$ and $y_{2}$ were defined in (4.4). The solution to (4.22) is given in terms of some unknown constant $A_{j}$ by

$$
\Psi=\mu_{j} A_{j} \log \left(|y| / \beta_{j}\right), \quad \mu_{j} \equiv-\frac{1}{\log \left(\varepsilon \beta_{j}\right)}, \quad \beta_{j} \equiv a_{j} \exp \left(-1 / a_{j} \kappa_{j}\right) .
$$

To formulate a problem that has the effect of summing all of the logarithmic terms in the asymptotic expansion of (2.5), we do not expand $\psi$ or $\sigma(\varepsilon)$ in a logarithmic series as in (4.2) and (4.1). Instead, we take the outer solution for $\psi$ to satisfy (2.5a) in the punctured domain $S \backslash\left\{x_{1}, \ldots, x_{N}\right\}$, with a singularity behavior at each $x_{j}$ that asymptotically matches with (4.23). In this way, the hybrid asymptotic-numerical formulation for the outer solution for $\psi$ and the eigenvalue $\sigma(\varepsilon)$ is to solve

$$
\begin{gathered}
\triangle_{s} \psi+\sigma \psi=0, \quad x \in S \backslash\left\{x_{1}, \ldots, x_{N}\right\}, \\
\psi \sim A_{j}+\mu_{j} A_{j} \log \left|x-x_{j}\right| \text { as } \quad x \rightarrow x_{j},
\end{gathered}
$$

with $\int_{S} \psi^{2} d s=1$, where $\psi$ is singularity-free at the poles $\theta=0, \pi$ and is $2 \pi$ periodic in $\phi$. 
The solution to (4.24) is written in terms of the Helmholtz Green's function of (A.7), with $\sigma=\nu(\nu+1)$ in (A.7a), as

$$
\psi=-2 \pi \sum_{i=1}^{N} \mu_{i} A_{i} G_{h}\left(x ; x_{i}\right) .
$$

Then, by using (A.11a) of Principal Result A.1 of Appendix A, we can expand $\psi$ as $x \rightarrow x_{j}$ to obtain that

$$
\psi \sim \mu_{j} A_{j} \log \left|x-x_{j}\right|-2 \pi \mu_{j} A_{j} R_{h}-2 \pi \sum_{\substack{i=1 \\ i \neq j}}^{N} \mu_{i} A_{i} G_{h j i} \quad \text { as } \quad x \rightarrow x_{j} .
$$

By comparing (4.26) with the required singular behavior (4.24b), we conclude that $A_{j}$ must satisfy the following homogeneous linear system:

$$
A_{j}+2 \pi \mu_{j} A_{j} R_{h}+2 \pi \sum_{\substack{i=1 \\ i \neq j}}^{N} \mu_{i} A_{i} G_{h j i}=0, \quad j=1, \ldots, N .
$$

Here $G_{h j i}=G_{h}\left(x_{j} ; x_{i}\right)$ is given in terms of $\sigma=\nu(\nu+1)$ by (A.8). The condition that this system has a nontrivial solution determines the eigenvalue $\sigma(\varepsilon)$, while the corresponding eigenvector determines $A_{1}, \ldots, A_{N}$ up to a scalar multiple. This scalar multiple can then be found by substituting (4.25) into the normalization condition $\int_{S} \psi^{2} d s=1$. This leads to the following result.

PRINCIPAL RESUlt 4.2. Consider (2.5) for $N$ partially absorbing traps of radii $\varepsilon a_{j}$ for $j=1, \ldots, N$. Then, with an error of order $\mathcal{O}(\varepsilon)$, the principal eigenvalue $\sigma(\varepsilon)$ of $(2.5)$ is the smallest root of the transcendental equation

$$
\operatorname{Det}\left(I+2 \pi R_{h} \mathcal{U}+2 \pi \mathcal{G}_{h} \mathcal{U}\right)=0 .
$$

Here $\mathcal{U}$ is the diagonal matrix with diagonal entries $\mathcal{U}_{j j}=\mu_{j}$ for $j=1, \ldots, N$, and $\mathcal{G}_{h}$ is the Helmholtz Green's function matrix with matrix entries

$$
\mathcal{G}_{h j j}=0, \quad j=1, \ldots, N ; \quad \mathcal{G}_{h i j}=-\frac{1}{4 \sin (\pi \nu)} P_{\nu}\left(\frac{\left|x_{j}-x_{i}\right|^{2}}{2}-1\right), \quad i \neq j,
$$

where $P_{\nu}(z)$ is the Legendre function of the first kind of order $\nu$ (see (A.8)).

We remark that both $R_{h}$, given in (A.11b), and $\mathcal{G}_{h i j}$ depend on $\nu$. In section 5 we solve (4.28) numerically for $\nu=\nu(\varepsilon)$, which determines $\sigma(\varepsilon)$ from $\sigma=\nu(\nu+1)$. For the special case of identical perfectly absorbing traps where $\mathcal{U}=\mu I$ with $\mu=-1 / \log (\varepsilon a)$, the solution to (4.28) extends the two-term result in Principal Result 4.1 by effectively summing all of the logarithmic terms in the expansion of $\sigma(\varepsilon)$.

For the special case of one trap $N=1,(4.28)$ reduces to the transcendental equation $2 \pi R_{h}=-1 / \mu_{1}$. By using (A.11b) for $R_{h}$, we obtain that $\sigma(\varepsilon)$ is the smallest root of

$$
-\log 2+\gamma+\psi(\nu+1)+\frac{\pi}{2} \cot (\pi \nu)=\frac{1}{\mu_{1}}, \quad \sigma=\nu+\nu^{2},
$$

where $\gamma$ is Euler's constant and $\psi(z)$ is the digamma function defined in (A.10). We can readily recover the two-term expansion (4.18) by substituting $\psi(\nu+1) \sim-\gamma$ 
and $\pi \cot \pi \nu \sim 1 / \nu$ for $\nu \ll 1$ into (4.29) and then solving for $\sigma$. For a perfectly absorbing trap of radius $\varepsilon$, in Figure 3 we compare the result for $\sigma(\varepsilon)$ obtained from the transcendental equation (4.29) with that given by the two-term result (4.18). Since $\pi \varepsilon^{2} /(4 \pi)=(\varepsilon / 2)^{2}=0.04$ when $\varepsilon=0.4$, we conclude from this figure that the two-term expansion provides a decent approximation to the eigenvalue provided that the trap area is less than roughly $4 \%$ of the surface area of the unit sphere.

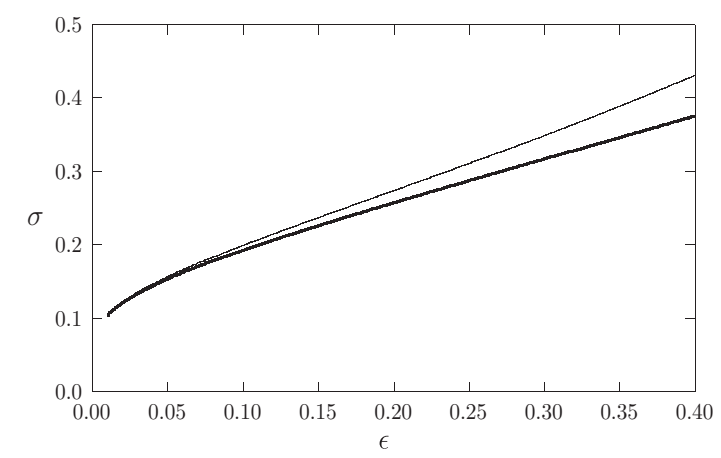

FIG. 3. For one perfectly absorbing trap of radius $\varepsilon$, we compare the two-term expansion for $\sigma(\varepsilon)$ given in (4.18) (top curve) with the result obtained from the transcendental equation (4.29) (bottom curve), which sums all logarithmic terms.

Next, we use (4.28) to derive a two-term expansion for $\sigma(\varepsilon)$ for the case of partially absorbing traps. For $\nu \rightarrow 0$, we use (A.14) for $G_{h}$ to write $\mathcal{G}_{h}$ as

$$
\mathcal{G}_{h}=-\frac{1}{2 \pi} \mathcal{G}_{0}+N R_{h 0} E-R_{h 0} I, \quad E \equiv \frac{1}{N} e e^{t} .
$$

Here $e^{t}=(1, \ldots, 1), R_{h 0}$ is given in (A.15), and $\mathcal{G}_{0}$ is the Green's function matrix of Principal Result 3.1 with zero diagonal entries and off-diagonal entries $\mathcal{G}_{0 i j}=$ $\log \left|x_{i}-x_{j}\right|$ for $i \neq j$. Upon using (4.30), we can write (4.27) asymptotically as a matrix eigenvalue problem of the form

$$
\mathcal{B} \mathcal{A}=\omega \mathcal{A}, \quad \mathcal{B} \equiv\left(I-\mathcal{G}_{0} \mathcal{U}\right)^{-1} E \mathcal{U}, \quad \omega \equiv-\frac{1}{2 \pi N R_{h 0}} .
$$

Since $E$ is a matrix of rank one and $\mathcal{U}$ is invertible, then $\mathcal{B}$ also has rank one. Hence the unique nonzero eigenvalue of $\mathcal{B}$ is $\omega=\operatorname{Trace}(B)$. Hence, for $\mu_{j} \ll 1$, we obtain the transcendental equation

$$
-\frac{1}{2 \pi N R_{h 0}}=\operatorname{Trace}\left[\left(I-\mathcal{G}_{0} \mathcal{U}\right)^{-1} E \mathcal{U}\right]
$$

where the right-hand side of (4.32) is independent of $\nu$.

For $\nu \ll 1$ and $\mu_{j} \ll 1$ for $j=1, \ldots, N$, we use (A.15) for $R_{h 0}$ together with the approximate inverse $\left(I-\mathcal{G}_{0} \mathcal{U}\right)^{-1} \approx I+\mathcal{G}_{0} \mathcal{U}$ to reduce (4.32) to

$$
\begin{aligned}
\frac{2 \nu}{N(1-2 \nu \log 2)} & \sim \operatorname{Trace}(E \mathcal{U})+\operatorname{Trace}\left[\mathcal{G}_{0} \mathcal{U} E \mathcal{U}\right]+\cdots, \\
\frac{1}{N}\left[2 \nu+4 \nu^{2} \log 2\right] & \sim \bar{\mu}+\operatorname{Trace}\left[\mathcal{G}_{0} \mathcal{U} E \mathcal{U}\right]+\cdots .
\end{aligned}
$$

Copyright $\odot$ by SIAM. Unauthorized reproduction of this article is prohibited. 
We then solve this equation asymptotically for $\nu$ and we identify that Trace $\left[\mathcal{G}_{0} \mathcal{U} E \mathcal{U}\right]=$ $2 p_{w}\left(x_{1}, \ldots, x_{N}\right)$, where $p_{w}\left(x_{1}, \ldots, x_{N}\right)$ is the weighted discrete energy of (3.31c). In this way, we obtain the following two-term expansion.

Principal Result 4.3. Let $\sigma(\varepsilon)$ be the principal eigenvalue of $(2.5)$ for $N$ partially absorbing traps of radii $\varepsilon a_{j}$ for $j=1, \ldots, N$. Then, a two-term logarithmic expansion for $\sigma(\varepsilon)$ is given explicitly by

$$
\sigma(\varepsilon) \sim \frac{N \bar{\mu}}{2}-\frac{(N \bar{\mu})^{2}}{4}(2 \log 2-1)+p_{w}\left(x_{1}, \ldots, x_{N}\right)+\mathcal{O}\left(|\mu|^{3}\right),
$$

where $\bar{\mu}$ is defined by

$$
\bar{\mu} \equiv \frac{1}{N} \sum_{j=1}^{N} \mu_{j}, \quad \mu_{j} \equiv-\frac{1}{\log \left(\varepsilon \beta_{j}\right)}, \quad \beta_{j} \equiv a_{j} \exp \left(-1 / a_{j} \kappa_{j}\right),
$$

and $p_{w}\left(x_{1}, \ldots, x_{N}\right)$ is the weighted discrete energy given in (3.31c).

For the special case of one circular partially absorbing trap of radius $\varepsilon,(4.33)$ with $N=1$ and $a_{1}=1$ reduces to

$$
\sigma(\varepsilon) \sim \frac{\mu}{2}+\mu^{2}\left(\frac{1}{4}-\frac{\log 2}{2}\right)+\cdots, \quad \mu=\left(-\log \left[\varepsilon e^{-1 / \kappa_{1}}\right]\right)^{-1} .
$$

This result agrees with the result (2.11) of section 2, as originally derived in [47].

Finally, we remark that noncircular traps can be treated simply by replacing $\beta_{j}$ in (4.34) with $d_{j}\left(\kappa_{j}\right)$, where $d_{j}\left(\kappa_{j}\right)$ is to be computed from the modified inner problem (3.33). For a cluster of traps in an $\mathcal{O}(\varepsilon)$ neighborhood of some point on the sphere, $d_{j}$ is to be found from (3.34).

5. Numerical results and validation of asymptotic theory. In this section we compare the asymptotic results for the solutions of (2.1) and (2.5) for perfectly (3.1) and partially (3.25) absorbing traps. In (2.1) we will consider two cases for the source term:

$$
M_{1} \equiv 1 \quad \text { and } \quad M_{2}=\frac{1}{2}(1+\cos 2 \theta) .
$$

The choice $M_{1}$ corresponds to a constant source density which yields the mean first passage time for perfectly absorbing traps, i.e., $b_{j}=0$ for $j=1, \ldots, N$ in (3.1). The second choice with $M_{2}$ models a source density that is largest at the poles and zero on the equatorial plane. In the case of $M_{1}$ the solution of (3.11) is simply $u_{p} \equiv 0$, while for $M_{2}$ it is given by $u_{p}=(1 / 6)\left(\cos ^{2} \theta-1 / 3\right)$.

5.1. Steady state diffusion. To compare the asymptotic solution (3.10) with those from numerical simulations using the COMSOL software package [8], we must solve (3.17) in the case of perfect traps and (3.30) for partially absorbing traps. Figure 4 shows the numerical solution of (3.1) with $b_{j}=0$ for a 5 -trap configuration that maximizes the discrete energy $p\left(x_{1}, \ldots, x_{5}\right)$ defined in $(3.18 \mathrm{~d})$. This optimal configuration has a trap at each pole together with three traps forming an equilateral triangle on the equatorial plane (cf. [11]). For the inhomogeneous source distribution $M_{2}$ in (5.1) the mean first passage time $u$ is maximal in the two belt-like regions along the $\phi$-direction (Figure 4(a)), while for the homogeneous distribution $M_{1}$ it is maximal in the three rectangular regions between each of the two equatorial traps 

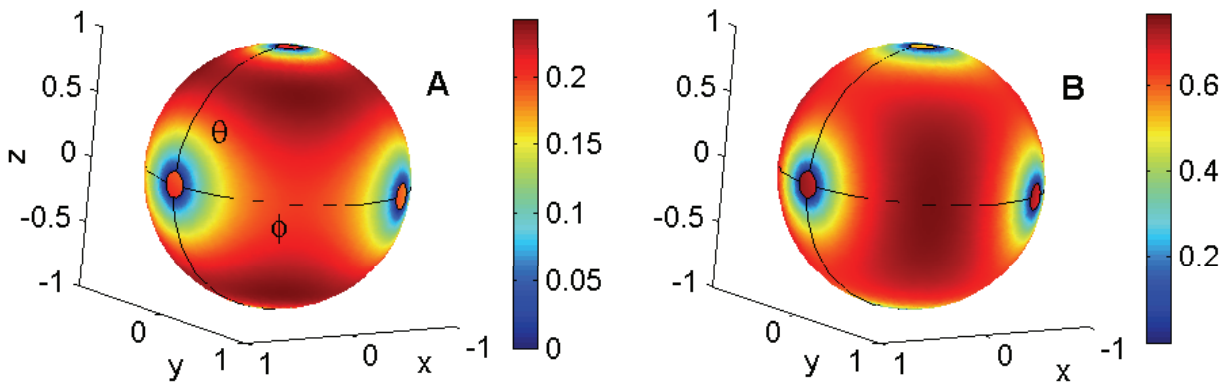

FIG. 4. Solution of (2.1) with $M_{2}=(1 / 2)(1+\cos 2 \theta)$ (a) and $M_{1}=1$ (b) for a 5-trap configuration with $\left(\theta_{1}, \phi_{1}\right)=(0,0),\left(\theta_{2}, \phi_{2}\right)=(\pi, 0),\left(\theta_{3}, \phi_{3}\right)=(\pi / 2,0),\left(\theta_{4}, \phi_{4}\right)=(\pi / 2,2 \pi / 3)$, and $\left(\theta_{5}, \phi_{5}\right)=(\pi / 2,4 \pi / 3)$ that maximizes the discrete energy $p\left(x_{1}, \ldots, x_{5}\right)$ as defined in $(3.18 \mathrm{~d})$. The traps are identical and perfectly absorbing, and have a common radius $\varepsilon=0.1$.
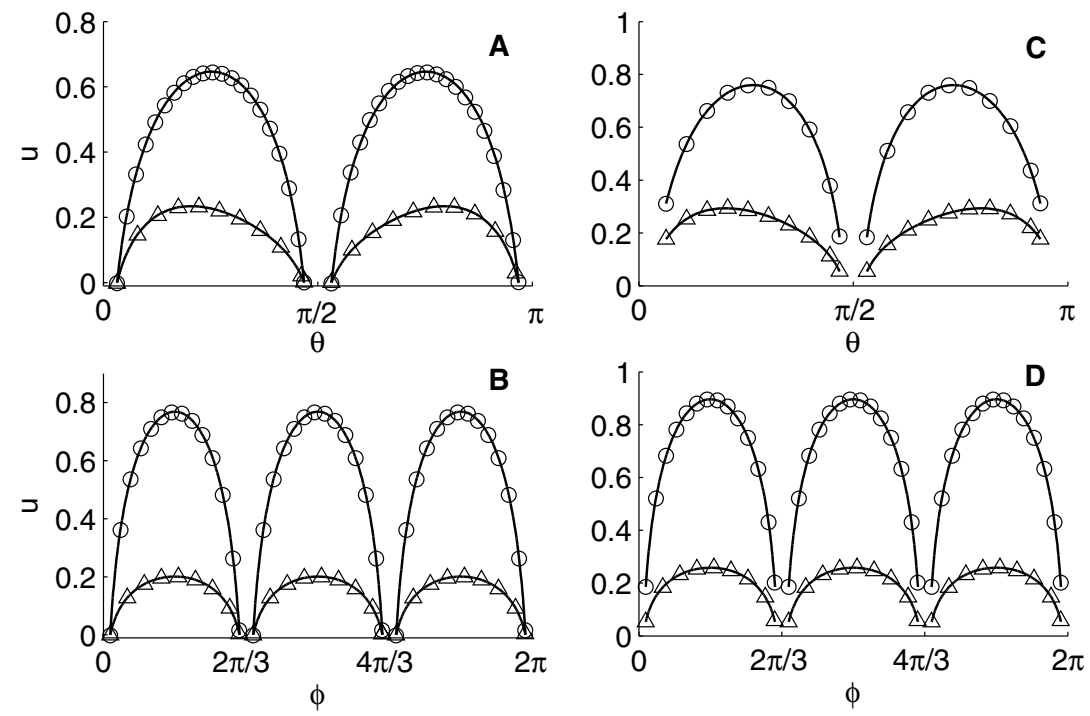

FIG. 5. Comparison between numerical solutions (solid lines) of (2.1) and the asymptotic results given by (3.10), (3.17), and (3.30) for perfectly (a), (b) and partially (c), (d) absorbing traps in the 5-trap configuration shown in Figure 4. (a) and (c) show the solution at $\phi=0$, while (b) and (d) show it along the equator $\theta=\pi / 2$. Open circles and triangles correspond to asymptotic solutions with $M_{1}=1$ and $M_{2}=(1 / 2)(1+\cos 2 \theta)$ in (2.1), respectively. Parameters for (a), $(\mathrm{b}): b_{i}=0$, $a_{i}=1$ for $i=1, \ldots, 5, \varepsilon=0.1$. Parameters for (c), (d): $b_{1}=0.1=b_{2}, b_{3}=0=b_{4}=b_{5}$, $a_{1}=1=a_{2}, a_{3}=0.5=a_{4}=a_{5}, \varepsilon=0.2, \kappa_{1}=2=\kappa_{2}, \kappa_{3}=4=\kappa_{4}=\kappa_{5}$.

(Figure 4(b)). In Figure 5 we compare the numerical solutions (solid lines) shown in Figure 4 along the longitudinal direction for fixed $\phi=0$ (Figure 5(a)) and along the latitudinal direction for $\theta=\pi / 2$ (Figure 5(b)) with the asymptotic result (3.17). In the computations we have taken identical, perfectly absorbing traps with a common radius $\varepsilon=0.1$, which corresponds to $\mu=-1 / \log \varepsilon \approx 0.43$. Although this value of $\mu$ is not small compared with unity as should be required by the asymptotic theory of section 3 , the asymptotic solutions are still found to be in excellent agreement with the full numerical solutions computed from COMSOL. 

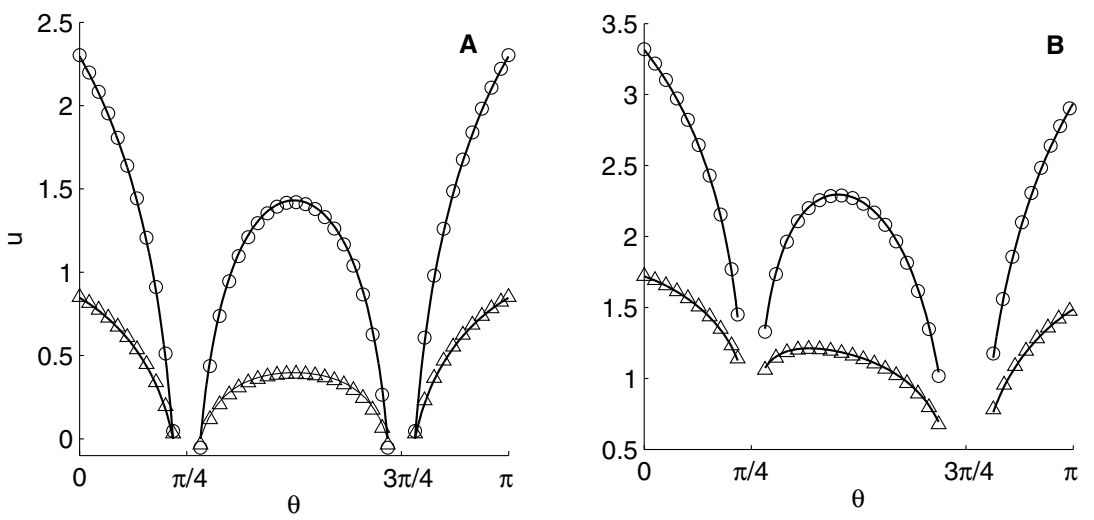

FIG. 6. Comparison between numerical solutions (solid lines) of (2.1) and the asymptotic results given in (3.10), (3.17), and (3.30) for perfectly (a) and partially (b) absorbing traps in a nonsymmetrical 2-trap configuration with $\left(\theta_{1}, \phi_{1}\right)=(\pi / 4,0)$ and $\left(\theta_{2}, \phi_{2}\right)=(3 \pi / 4,0)$. Shown are solutions at $\phi=0$. Open circles and triangles correspond to asymptotic solutions with $M_{1}=1$ and $M_{2}=(1 / 2)(1+\cos 2 \theta)$ in $(2.1)$, respectively. Parameters for $(\mathrm{a}): b_{1}=0=b_{2}, \varepsilon=0.1$. Parameters for (b): $b_{1}=1, b_{2}=0.5, a_{1}=0.5, a_{2}=1, \varepsilon=0.2, \kappa_{1}=4, \kappa_{2}=2$.

In Figures 5(c) and 5(d) we show a similar comparison between the full numerical and the asymptotic solutions (3.30) for partially absorbing traps of different radius. Here, the radii of the largest traps are $\varepsilon=0.2$, which corresponds $\mu=-1 / \log \varepsilon \approx$ 0.62 . Even with this relatively large value of $\mu$, the asymptotic results still agree extremely well with the full numerical solutions. We mention that we would have obtained the same results if we had used the asymptotic approximations for $\mathcal{A}$ and $\chi$ in (3.18b), (3.31a) and (3.18c), (3.31b), respectively. Upon increasing the radius of the trap we observed that the asymptotic solutions begin to diverge from the numerical ones at $\varepsilon=0.2(\varepsilon=0.3)$ in the case of perfectly (partially) absorbing traps for the 5 -trap configuration.

In Figure 5, the excellent agreement between the asymptotic solutions and those from full numerical simulations is partially due to the particular configuration of the traps. For example, Figure 6 shows a 2-trap configuration where a small deviation becomes visible at the boundary of the traps (Figure 6(a)) as the zero boundary condition is not exactly satisfied. For partially absorbing traps in the same configuration the deviation is smaller (Figure 6(b)).

5.2. Eigenvalue asymptotics. We have used the eigenvalue solver of the COMSOL software package [8] to compute the smallest eigenvalue together with the corresponding eigenmode of (2.5) for the 2- and 5-trap configurations shown in Figures 6(a) and 5(a) for identical perfectly absorbing traps. Figure 7 shows the numerical results (solid lines) together with the asymptotic solutions in (4.2) (dashed lines) and (4.25) (open circles). For the 2-trap configurations (Figures 7(a) and 7(b)) the two-term expansion yields a reasonable approximation even for $\varepsilon=0.1$ (see Figure $5(\mathrm{~b})$ ). In contrast, for the 5-trap configuration the two-term expansion fails to correctly reproduce the absorbing boundary conditions even at $\varepsilon=0.05$. In contrast, the asymptotic solution (4.25), which sums all logarithmic corrections, is in excellent agreement with the numerical solution up to $\varepsilon=0.1$. Table 1 shows the smallest eigenvalue as a function of the trap radius $\varepsilon$ and compares it with the asymptotic results from (4.28a) and (4.17). The corresponding results are shown graphically in Figure 8. 

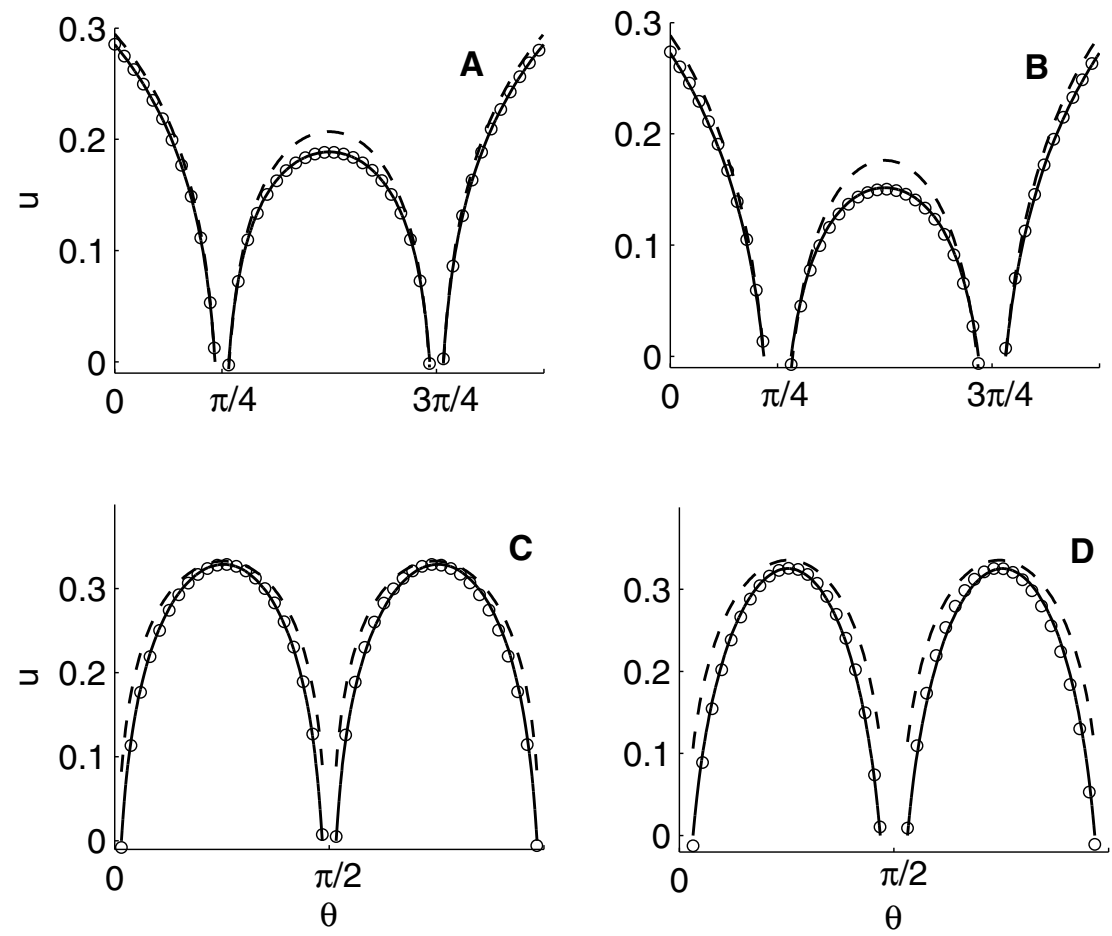

FIG. 7. Eigenmodes corresponding to the smallest eigenvalue of (2.5) for the 2- (a), (b) and 5- (c), (d) (perfect) trap configurations shown in Figures 6(a) and 5(a). Solid lines are numerical solutions. Open circles mark the asymptotic solution in (4.25), while the dashed lines correspond to the two-term expansion in (4.2) with $\psi_{0}=1 /(4 \pi)$ and $\psi_{1}$ given by (4.11). In (a), (c) $\varepsilon=0.05$, while in (b), (d) $\varepsilon=0.1$.

TABLE 1

Smallest eigenvalue of (2.5) for the 2- and 5-trap configurations shown in Figures 6(a) and 5(a) as a function of the trap radius $\varepsilon$. Here, $\sigma$ is the numerical solution found by COMSOL [8], $\sigma^{*}=$ $\nu(1+\nu)$ corresponds to the root $\nu$ of the transcendental equation $(4.28 \mathrm{a})$, and $\sigma_{2}$ is calculated from the two-term expansion (4.17).

\begin{tabular}{l|ccc|ccc}
\hline & \multicolumn{3}{|c|}{5 traps } & \multicolumn{3}{c}{2 traps } \\
\hline$\varepsilon$ & $\sigma$ & $\sigma^{*}$ & $\sigma_{2}$ & $\sigma$ & $\sigma^{*}$ & $\sigma_{2}$ \\
\hline 0.02 & 0.7918 & 0.7894 & 0.7701 & 0.2458 & 0.2451 & 0.2530 \\
0.05 & 1.1003 & 1.0991 & 1.0581 & 0.3124 & 0.3121 & 0.3294 \\
0.1 & 1.5501 & 1.5452 & 1.4641 & 0.3913 & 0.3903 & 0.4268 \\
0.2 & 2.5380 & 2.4779 & 2.3278 & 0.5177 & 0.5110 & 0.6060 \\
\hline
\end{tabular}

Finally, we illustrate the effect of the locations of the traps on the sphere with regard to the eigenvalue approximation $\sigma(\varepsilon)$, computed from (4.28a), and the averaged mean first passage time $\chi$, computed from (3.17c) for the special case $M=1$ and $f=0$ in $(3.17 \mathrm{c})$. We consider three different spatial configurations of four identical perfectly absorbing traps with a common radius $\varepsilon$. The specific configurations and the corresponding asymptotic results for $\sigma(\varepsilon)$ and $\chi(\varepsilon)$ are shown in Figures 9(a) and $9(\mathrm{~b})$, respectively, and are compared there with full numerical results computed 


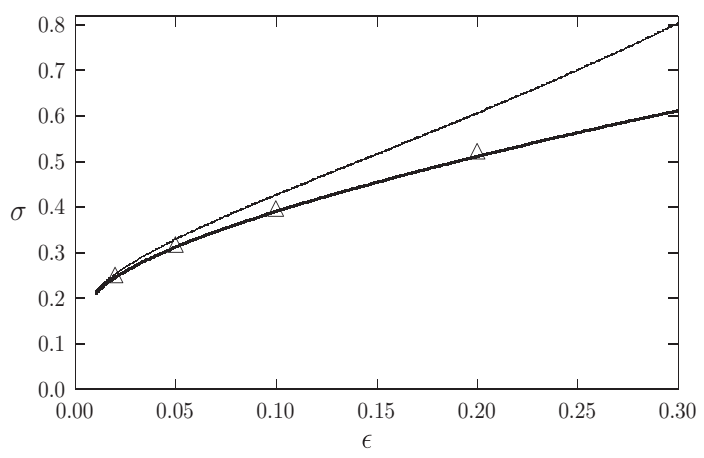

(a) 2-trap pattern

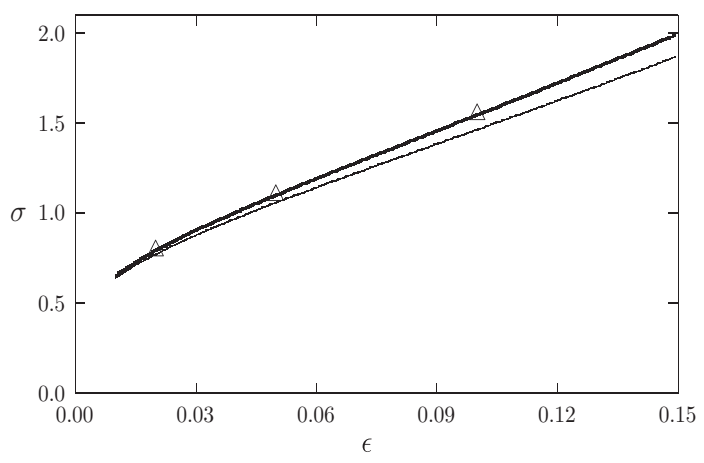

(b) 5-trap pattern

FIG. 8. Comparison of the two-term approximation (solid curve) for $\sigma(\varepsilon)$ versus $\varepsilon$ given in (4.17) with the root of the transcendental equation (4.28a) (heavy solid curve) for the 2-trap configuration (top) and the 5-trap configuration (bottom) shown in Figures 6(a) and 5(a), respectively. The data at selected points are given in Table 1.

from COMSOL [8]. Notice that the optimal 4-trap pattern, with two traps at the poles and two traps equally spaced on the equatorial plane, is the configuration that gives the largest $\sigma(\varepsilon)$ and the smallest averaged mean first passage time $\chi(\varepsilon)$. Such a 4 -trap pattern, with widely spaced traps, is optimal for minimizing the lifetime of a random walker on the sphere.

6. Discussion and conclusion. We have given asymptotic results for the mean first passage time for a diffusing particle confined to a spherical surface that has $N$ partially absorbing traps of asymptotically small radii. By asymptotically calculating the principal eigenvalue for diffusion on the sphere with small localized traps, we have determined analytically the asymptotic rate at which a small diffusing molecule becomes captured by one of the small traps. The asymptotic results were favorably compared with full numerical results computed from COMSOL [8].

In the introduction we motivated our model using examples from cell biology. Using physical parameters we can now use our results to estimate the minimum time it takes for a surface-bound molecule to reach a molecular cluster on a spherical cell, for example. We take the diffusion coefficient of a typical surface molecule (e.g., LAT) to be $0.25 \mu \mathrm{m}^{2} / \mathrm{s}$ and consider $N$ nascent signaling regions (traps) of radius $10 \mathrm{~nm}$ on a cell of radius $5 \mu \mathrm{m}$. Using (3.23) we can place an approximate lower bound on the averaged mean first passage time (valid when $N$ is sufficiently large) for the 


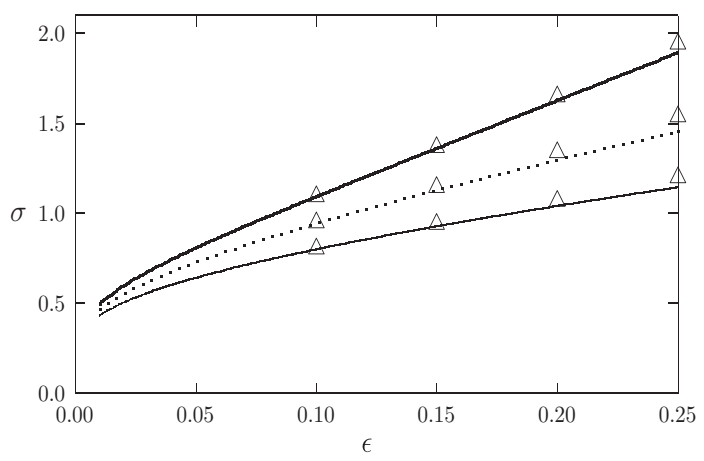

(a) $\sigma(\varepsilon)$ vs. $\varepsilon$

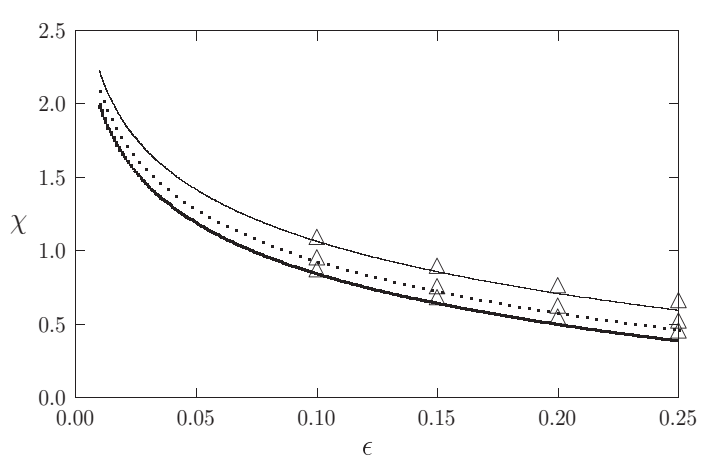

(b) $\chi(\varepsilon)$ vs. $\varepsilon$

FIG. 9. Results for $\sigma(\varepsilon)$, computed from (4.28a) (top) and $\chi(\varepsilon)$ computed from (3.17c) with $f=0$ and $M=1$ (bottom) for three different 4-trap patterns. The traps are perfectly absorbing and have a common radius $\varepsilon$. The heavy solid curves correspond to $\left(\theta_{1}, \phi_{1}\right)=(0,0),\left(\theta_{2}, \phi_{2}\right)=(\pi, 0)$, $\left(\theta_{3}, \phi_{3}\right)=(\pi / 2,0),\left(\theta_{4}, \phi_{4}\right)=(\pi / 2, \pi)$; the solid curves correspond to $\left(\theta_{1}, \phi_{1}\right)=(0,0),\left(\theta_{2}, \phi_{2}\right)=$ $(\pi / 3,0),\left(\theta_{3}, \phi_{3}\right)=(2 \pi / 3,0),\left(\theta_{4}, \phi_{4}\right)=(\pi, 0)$; and the dotted curves correspond to $\left(\theta_{1}, \phi_{1}\right)=(0,0)$, $\left(\theta_{2}, \phi_{2}\right)=(2 \pi / 3,0),\left(\theta_{3}, \phi_{3}\right)=(\pi / 2, \pi),\left(\theta_{4}, \phi_{4}\right)=(\pi / 3, \pi / 2)$. The marked points are the full numerical results computed from COMSOL [8].

diffusive arrival of a particular molecule to a cluster. For $N=100$ traps, the bound is $7.7 \mathrm{~s}$. This bound is achieved when the clusters are placed at the Fekete points on the sphere. Note that we can also compute the principal eigenvalue using (4.21). As a comparison, if the 100 traps were joined together to form one big trap of the same area, (2.8) gives the averaged mean first passage time as 360s. More generally, (3.21) allows this estimate to be made for any arbitrary distribution of separated traps that might be observed in experiments. Assuming that the signaling molecules are initially uniformly spread across the cell, these results can quantify the ability of cells to control the signaling rate at clusters by modulating their spatial distribution.

We close by outlining a few possible extensions of this study. One possible generalization of our analysis would be to consider a nonspherical surface, with possibly a spatially inhomogeneous surface diffusivity. Such an analysis would require detailed properties of the Green's function for the Laplace-Beltrami operator and the Helmholtz operator on the nonspherical surface. For a general surface these functions can only be determined numerically.

In the context of a spherical surface, a second natural extension of the work presented here would be to include chemical reactions within each signaling region 
(trap), combined with a detailed account of the escape of molecules from each trap. A simple problem of this nature was considered in [12], where, in the context of $\mathrm{T}$ cell activation, the delivery of surface-bound antigen to reactive signaling clusters was considered. In that study, diffusing antigen underwent binding and unbinding events within each cluster (bound antigen was assumed to be immobile), and the escape time from a disk-shaped region on a flat surface was calculated using matched asymptotics. It should be possible to analyze more complex situations of this nature, for instance, by finding the steady states of the system and examining their stability to small perturbations. The mathematical interest of such problems is that for the stability calculation the eigenvalue parameter would appear in both the differential operator and in the boundary condition on the boundary of each localized reaction site, effectively yielding a novel class of Steklov-type eigenvalue problems.

Finally, our analysis has been restricted to the steady-state situation where the traps are at fixed locations on the surface of the sphere. This is a considerable simplification of the biological situation where cell-surface regions may be expected to diffuse or undergo directed motion [45]. Furthermore, colliding traps can sometimes be expected to coalesce, while traps may also appear and disappear on the cell surface. Certain problems with moving traps have been analyzed, such as in [22] and [4], but the field remains quite open.

Appendix A. The modified and Helmholtz Green's functions on the sphere. In this appendix we give some properties of the modified and Helmholtz Green's functions on the unit sphere that are central to the analysis in sections 3 and 4 . We first introduce the inner product $(u, v)$ over the unit sphere $S$ defined by

$$
(u, v) \equiv \int_{S} u v d s=\int_{0}^{2 \pi} \int_{0}^{\pi} u v \sin \theta d \theta d \phi .
$$

If $u$ and $v$ are $2 \pi$ periodic in $\phi$, then Lagrange's identity $\left(\triangle_{s} u, v\right)=\left(u, \triangle_{s} v\right)$ holds. The following uniquely determined, modified Green's function $G\left(x ; x_{0}\right)$ is central to the asymptotic analysis of (2.1) and (2.5):

$$
\triangle_{s} G=\frac{1}{4 \pi}-\delta\left(x-x_{0}\right), \quad x \in S,
$$

$G$ is $2 \pi$ periodic in $\phi$ and smooth at $\theta=0, \pi$,

$$
\int_{S} G d s \equiv \int_{0}^{2 \pi} \int_{0}^{\pi} G \sin \theta d \theta d \phi=0,
$$

where, in terms of spherical coordinates, $x$ and $x_{0}$ are given by (A.3)

$$
x=(\cos \phi \sin \theta, \sin \phi \sin \theta, \cos \theta), \quad x_{0}=\left(\cos \phi_{0} \sin \theta_{0}, \sin \phi_{0} \sin \theta_{0}, \cos \theta_{0}\right) .
$$

Since $\left(\triangle_{s} G, 1\right)=0$ the divergence theorem is satisfied.

The solution of (A.2) is well known from various studies of the motion of fluid vortices on the sphere (cf. [24], [25], and [26]) and is given explicitly by

$$
G\left(x ; x_{0}\right)=-\frac{1}{4 \pi}\left[\log \left(1-x \cdot x_{0}\right)+1-\log 2\right],
$$

where $\cdot$ denotes dot product. From the law of cosines, $1-x \cdot x_{0}=\left|x-x_{0}\right|^{2} / 2$, so that (A.4) becomes

$$
G\left(x ; x_{0}\right)=-\frac{1}{2 \pi} \log \left|x-x_{0}\right|+R, \quad R \equiv \frac{1}{4 \pi}[2 \log 2-1],
$$


where $R$ is the regular part of $G$. We then calculate the Jacobian of (A.3) to conclude that as $x \rightarrow x_{0}$

$$
\left|x-x_{0}\right|=|\hat{y}|+o(1), \quad \hat{y}=\left(\hat{y}_{1}, \hat{y}_{2}\right)=\left(\hat{\phi} \sin \theta_{0}, \hat{\theta}\right) .
$$

In section 4 we require some properties of the Helmholtz Green's function $G_{h}\left(x ; x_{0}\right)$, satisfying

$$
\begin{aligned}
& \triangle_{s} G_{h}+\nu(\nu+1) G_{h}=-\delta\left(x-x_{0}\right), \quad x \in S, \\
& G_{h} \text { is } 2 \pi \text { periodic in } \phi \text { and smooth at } \theta=0, \pi .
\end{aligned}
$$

This Green's function, which arises in the study of high frequency wave scattering (cf. [38]; see also [43]), is given explicitly by

$$
G_{h}\left(x ; x_{0}\right)=-\frac{1}{4 \sin (\pi \nu)} P_{\nu}\left(-x \cdot x_{0}\right),
$$

where $P_{\nu}(z)$ is the Legendre function of the first kind of order $\nu$. As $z \rightarrow-1$, it follows from [13] that

$$
P_{\nu}(z) \sim \frac{\sin (\pi \nu)}{\pi}\left[\log \left(\frac{1+z}{2}\right)+2 \gamma+2 \psi(\nu+1)+\pi \cot (\pi \nu)\right],
$$

where $\psi(z)$ is the psi or digamma function, which is defined in terms of the Gamma function $\Gamma(z)$ by (cf. [13])

$$
\psi(z)=\frac{\Gamma^{\prime}(z)}{\Gamma(z)}=\log z+\int_{0}^{\infty} e^{-z t}\left(\frac{1}{t}-\frac{1}{1-e^{-t}}\right) d t .
$$

Here $\gamma \approx 0.577216$ is Euler's constant. Upon substituting (A.9) into (A.8), and recalling that $1-x \cdot x_{0}=\left|x-x_{0}\right|^{2} / 2$, we readily obtain the following result required in section 4 .

Principal Result A.1. As $x \rightarrow x_{0}, G_{h}\left(x ; x_{0}\right)$ satisfies

$$
G_{h}\left(x ; x_{0}\right)=-\frac{1}{2 \pi} \log \left|x-x_{0}\right|+R_{h}(\nu)+o(1) \quad \text { as } \quad x \rightarrow x_{0},
$$

where

$$
R_{h}(\nu) \equiv-\frac{1}{4 \pi}[-2 \log 2+2 \gamma+2 \psi(\nu+1)+\pi \cot (\pi \nu)] .
$$

In section 4 we need a result for $G_{h}$ in the limit $\nu \rightarrow 0$ that is uniformly valid in $x$. For $\nu \rightarrow 0$, we expand the solution $G_{h}$ to (A.7) as

$$
G_{h}=\frac{1}{\nu} G_{-1}+G_{0}+\nu G_{1}+\cdots,
$$

where, on the unit sphere, $G_{-1}, G_{0}$, and $G_{1}$ solve

$$
\triangle_{s} G_{-1}=0, \quad \triangle_{s} G_{0}=-G_{-1}-\delta\left(x-x_{0}\right), \quad \triangle_{s} G_{1}=-G_{-1}-G_{0} .
$$

Thus, $G_{-1}$ is a constant, and from the equation for $G_{0}$, we get that $G_{-1}=-1 / 4 \pi$. From the equation for $G_{1}$, we obtain that $\int_{S} G_{0} d s=1$. Therefore, $G_{0}=G+1 /(4 \pi)$, 
where $G$ satisfies (A.2). Hence, by using (A.5) for $G$, we have for $\nu \ll 1$, and uniformly in $x$, that

$$
G_{h}=-\frac{1}{2 \pi} \log \left|x-x_{0}\right|+R_{h 0}+\mathcal{O}(\nu),
$$

where $R_{h 0}$ is defined by

$$
R_{h 0}=\frac{1}{4 \pi}\left[2 \log 2-\frac{1}{\nu}\right]+\mathcal{O}(\nu) \quad \text { as } \quad \nu \rightarrow 0 .
$$

We remark that the limiting form $R_{h 0}$ agrees with that obtained by substituting the limiting expressions $\psi(\nu+1) \sim-\gamma+\mathcal{O}(\nu)$ from [13]) and $\pi \cot \pi \nu \sim \nu^{-1}$ into the expression (A.11b) for $R_{h}(\nu)$.

Appendix B. The logarithmic capacitance of a two-disk cluster. We derive the result (3.35) for the logarithmic capacitance $d$ of two circles of common radius $a$, where $2 l$, with $l>a$, denotes the distance between the centers of the circles. Since $d$ is invariant under coordinate rotations, we conveniently choose the centers of the circles to lie along the horizontal axis. For this special 2-trap cluster, the inner problem (3.34) with $y=\left(y_{1}, y_{2}\right)$, and in a more convenient notation, is

$$
\begin{aligned}
v_{y_{1} y_{1}}+v_{y_{2} y_{2}} & =0, \quad y \notin \Omega_{j}, \quad j=1,2 ; \quad v=0, \quad y \in \partial \Omega_{j}, \\
v & \sim \log |y| \quad \text { as } \quad|y|=\left(y_{1}^{2}+y_{2}^{2}\right)^{1 / 2} \rightarrow \infty,
\end{aligned}
$$

where $\Omega_{1}$ and $\Omega_{2}$ are the circles $\left(y_{1}+l\right)^{2}+y_{2}^{2}=a^{2}$ and $\left(y_{2}-l\right)^{2}+y_{2}^{2}=a^{2}$, respectively. The logarithmic capacitance $d$ is defined in terms of the solution to (B.1) by the farfield condition

$$
v-\log |y|=-\log d+o(1) \quad \text { as } \quad|y|=\left(y_{1}^{2}+y_{2}^{2}\right)^{1 / 2} \rightarrow \infty .
$$

To solve (B.1) we introduce bipolar coordinates $\xi$ and $\eta$ defined by

$$
y_{1}=\frac{\beta \sinh \xi}{\cosh \xi-\cos \eta}, \quad y_{2}=\frac{\beta \sin \eta}{\cosh \xi-\cos \eta} .
$$

Then, $|y| \rightarrow \infty$ corresponds to $\rho \equiv\left(\xi^{2}+\eta^{2}\right)^{1 / 2} \rightarrow 0$. From (B.2) we obtain $|y| \sim 2 \beta / \rho$ as $|y| \rightarrow \infty$. Therefore, the singularity condition in (B.1b) is equivalent to $v \sim-\log \rho$ as $\rho=\left(\xi^{2}+\eta^{2}\right)^{1 / 2} \rightarrow 0$. We then define $\xi_{c}$ and $\beta$ by

$$
l=\beta \operatorname{coth} \xi_{c}, \quad a=\frac{\beta}{\sinh \xi_{c}},
$$

so that, for $l>a, \beta$ and $\xi_{c}$ are given uniquely by

$$
\beta=\sqrt{l^{2}-a^{2}} ; \quad \cosh \left(\xi_{c}\right)=\frac{l}{a}, \quad \xi_{c}=\log \left[\frac{l}{a}+\sqrt{\left(\frac{l}{a}\right)^{2}-1}\right] .
$$

Under the coordinate transformation the circle $\left(y_{1}-l\right)^{2}+y_{2}^{2}=a^{2}$ corresponds to $\xi=\xi_{c}$ with $|\eta| \leq \pi$. Similarly, the circle with center at $(-l, 0)$ corresponds to $\xi=-\xi_{c}$ with $|\eta| \leq \pi$. In terms of bipolar coordinates, (B.1) transforms to the following 
Green's function problem for $U(\xi, \eta) \equiv v\left(y_{1}(\xi, \eta), y_{2}(\xi, \eta)\right)$ in the rectangle $|\xi| \leq \xi_{c}$ and $|\eta| \leq \pi$ :

$$
\begin{gathered}
U_{\xi \xi}+U_{\eta \eta}=0, \quad|\xi| \leq \xi_{c}, \quad|\eta| \leq \pi, \\
U=0 \quad \text { on } \quad \xi=\xi_{c}, \quad \xi=-\xi_{c} ; \quad U, U_{\eta} \quad 2 \pi \text { periodic in } \eta, \\
U \sim-\log \rho+R+o(1) \quad \text { as } \quad \rho=\left(\xi^{2}+\eta^{2}\right)^{1 / 2} \rightarrow 0 .
\end{gathered}
$$

This problem uniquely defines the Green's function $U$ and its constant regular part $R$ at the origin. Upon using $\rho \sim 2 \beta /|y|$ as $\rho \rightarrow 0$ and $|y| \rightarrow \infty$, the singularity condition for $U$ transforms to $v \sim \log |y|-\log (2 \beta)+R$ as $|y| \rightarrow \infty$. Upon comparing this expression with (B.1c), we conclude that $d$ is related to $\beta=\left(l^{2}-a^{2}\right)^{1 / 2}$ and $R$ by

$$
\log d=\log (2 \beta)-R \text {. }
$$

To solve (B.4) we could write an eigenfunction expansion solution for $U$, similar to that in [40], and then use Ewald-type summation formulae to extract the regular part of the singularity behavior. Instead, we proceed in a more direct manner by first seeking a solution to Laplace's equation that is $2 \pi$ periodic in $\eta$ and that satisfies the singularity behavior in (B.4c). To this end, we define the harmonic function $H(\xi, \eta)$ for $\xi>0$ by

$$
\begin{aligned}
H & \equiv \sum_{m=1}^{\infty} \frac{e^{-m \xi}}{m} \cos (m \eta)=\operatorname{Re}\left[\sum_{m=1}^{\infty} \frac{\omega^{n}}{n}\right]=-\log |1-\omega| \\
& =-\frac{1}{2} \log \left(1-2 e^{-\xi} \cos \eta+e^{-2 \xi}\right),
\end{aligned}
$$

where $\omega \equiv e^{-(\xi+i \eta)}$ is the complex number with $\xi>0$. In (B.6) we used the identity $\sum_{m=1}^{\infty} m^{-1} \omega^{m}=-\log (1-\omega)$. As $\rho=\left(\xi^{2}+\eta^{2}\right)^{1 / 2} \rightarrow 0$, we get that $H \sim-\log \rho$, which yields the logarithmic singularity in (B.4c).

Next, we add a harmonic function to $H$ so that the resulting function is even in $\xi$. Since $H$ can be written as

$$
H=-\frac{1}{2} \log 2+\frac{\xi}{2}-\frac{1}{2} \log (\cosh \xi-\cos \eta),
$$

it follows that the harmonic function $U_{p}$ defined by

$$
U_{p}=H-\frac{\xi}{2}=-\frac{\xi}{2}+\sum_{m=1}^{\infty} \frac{e^{-m \xi}}{m} \cos (m \eta), \quad \xi>0,
$$

is $2 \pi$ periodic in $\eta$, is even in $\xi$, and satisfies the singularity condition in (B.4c). Therefore, we decompose the solution to (B.4) as $U=U_{p}+U_{h}$, where $U_{h}$ is harmonic with no singularities, is an even function of $\xi$, is $2 \pi$ periodic in $\eta$, and satisfies $U_{h}=$ $-U_{p}$ on $\xi=\xi_{c}$. This problem for $U_{h}$ is readily solved by separation of variables, to obtain that

$$
U=U_{p}+\frac{\xi_{c}}{2}-\sum_{m=1}^{\infty} \frac{1}{m} \frac{\cosh (m \xi)}{\cosh \left(m \xi_{c}\right)} e^{-m \xi_{c}} \cos (m \eta) .
$$

Finally, upon taking the limit $(\xi, \eta) \rightarrow(0,0)$, (B.9) becomes

$$
U=-\log \rho+R+o(1) \quad \text { as } \quad \rho \rightarrow 0, \quad R \equiv \frac{\xi_{c}}{2}-\sum_{m=1}^{\infty} \frac{e^{-m \xi_{c}}}{m \cosh \left(m \xi_{c}\right)} .
$$

Copyright $\odot$ by SIAM. Unauthorized reproduction of this article is prohibited. 
Therefore, the logarithmic capacitance $d$ from (B.5) is given by

$$
\log d=\log (2 \beta)-\frac{\xi_{c}}{2}+\sum_{m=1}^{\infty} \frac{e^{-m \xi_{c}}}{m \cosh \left(m \xi_{c}\right)}
$$

where $\beta$ and $\xi_{c}$ are determined in terms of the disk radius $a$ and half-separation $l$ by (B.3). This completes the derivation of (3.35) of Remark 3.2. As a remark, the analysis above is readily extended to calculate the logarithmic capacitance of two nonoverlapping disks of different radii.

\section{REFERENCES}

[1] N. Batada, L. Shepp, D. Siegmund, And M. Levitt, Spatial regulation and the rate of signal transduction activation, PLoS Comput. Biol., 2 (2006), pp. 0343-0349.

[2] H. C. Berg and E. M. Purcell, Physics of chemoreception, Biophys. J., 20 (1977), pp. 193219.

[3] B. Bergersen, D. Boal, and P. Palffy-Muhoray, Equilibrium configurations of particles on the sphere: The case of logarithmic interactions, J. Phys. A, 27 (1994), pp. 2579-2586.

[4] A. J. Bray And R. A. Blythe, Exact asymptotics for one-dimensional diffusion with mobile traps, Phys. Rev. Lett., 89 (2002), 150601.

[5] P. C. Bressloff, B. A. Earnshaw, and M. J. Ward, Diffusion of protein receptors on a cylindrical dendritic membrane with partially absorbing traps, SIAM J. Appl. Math., 68 (2008), pp. 1223-1246.

[6] G. Campi, R. Varma, And M. L. Dustin, Actin and agonist MHC-peptide complex-dependent $T$ cell receptor microclusters as scaffolds for signaling, J. Exp. Med., 202 (2005), pp. 10311036.

[7] N. Chao, S. H. Young, And M. Poo, Localization of cell membrane components by surface diffusion into a trap, Biophys. J., 36 (1981), pp. 139-153.

[8] COMSOL Multiphysics (v3.4), COMSOL AB, Stockholm, 2007.

[9] G. Del Grosso, A. Gerardi, and F. Marchetti, Lateral diffusion and eigenvalue estimates, Bull. Math. Biol., 45 (1983), pp. 627-634.

[10] W. Dijkstra and M. E. Hochstenbach, Numerical Approximation of the Logarithmic Capacity, preprint, CASA report 08-09, Technical University of Eindhoven, Eindhoven, The Netherlands, 2008.

[11] P. D. Dragnev, D. A. Legg, and D. W. Townsend, Discrete logarithmic energy on the sphere, Pacific J. Math., 207 (2002), pp. 345-358.

[12] O. DusheK And D. Coombs, Analysis of serial engagement and peptide-MHC transport in T cell receptor microclusters, Biophys. J., 94 (2008), pp. 3447-3460.

[13] A. Erdélyi, W. Magnus, F. Oberhettinger, and F. Tricomi, Higher Transcendental Functions, Vols. I and II, McGraw-Hill, New York, 1953.

[14] V. I. Fabrikant, Potential of several arbitrarily located disks, J. Austral. Math. Soc. Ser. B, 29 (1988), pp. 342-351.

[15] V. I. Fabrikant, Applications of Potential Theory in Mechanics: A Selection of New Results, Math. Appl. 51, Kluwer Academic Publishers, Dordrecht, The Netherlands, 1989.

[16] M. Feкете, Über die Verteilung der Wurzeln bei Gewissen Algebraischen Gleichungen mit ganzzahligen Koeffizienten, Math. Z., 17 (1923), pp. 228-249.

[17] A. Gandolfi, A. Gerardi, and F. Marchetti, Association rates of diffusion-controlled reactions in two dimensions, Acta Appl. Math., 4 (1985), pp. 139-155.

[18] S. Gemmrich, N. Nigam, and O. Steinbach, Boundary integral equations for the LaplaceBeltrami operator, in Mathematics and Computation, a Contemporary View, Abel Symposia 3, Springer, Berlin, Heidelberg, 2008, pp. 21-37.

[19] B. Goldstein, C. Wofsy, and H. Echavarria-Heras, Effect of membrane flow on the capture of receptors by coated pits. Theoretical results, Biophys. J., 53 (1988), pp. 405-414.

[20] D. Holcman and Z. Schuss, Escape through a small opening: Receptor trafficking in a synaptic membrane, J. Statist. Phys., 117 (2004), pp. 975-1014.

[21] D. Holcman And Z. Schuss, Diffusion escape through a cluster of small absorbing windows, J. Phys. A, 41 (2008), 155001

[22] F. Den Hollander and G. H. Weiss, Aspects of trapping in transport processes, in Contemporary Problems in Statistical Physics, G. H. Weiss, ed., SIAM, Philadelphia, 1994.

Copyright $@$ by SIAM. Unauthorized reproduction of this article is prohibited. 
[23] C. A. Janeway, P. Travers, M. Walport, and J. D. Capra, Immunobiology, Elsevier, London, UK, 1999.

[24] R. Kidambi And P. K. Newton, Motion of three point vortices on a sphere, Phys. D, 116 (1998), pp. 143-175.

[25] R. Kidambi And P. K. Newton, Point vortex motion on a sphere with solid boundaries, Phys. Fluids, 12 (2000), pp. 581-588.

[26] Y. Kimura and H. Окамото, Vortex motion on a sphere, J. Phys. Soc. Japan, 56 (1987), pp. 4203-4206.

[27] T. Kolokolnikov, M. Titcombe, and M. J. Ward, Optimizing the fundamental Neumann eigenvalue for the Laplacian in a domain with small traps, European J. Appl. Math., 16 (2005), pp. 161-200.

[28] A. V. Kolushov And V. A. Yudin, Extremal dispositions of points on the sphere, Anal. Math., 23 (1997), pp. 25-34.

[29] J. Linderman and D. A. Laufenberger, Analysis of intracellular receptor/ligand sorting: Calculation of mean surface and bulk diffusion times within a sphere, Biophys. J., 50 (1986), pp. 295-305.

[30] I. L. Novak, F. Gao, Y. Choi, D. Resasco, J. Schaff, and B. Slepchenko, Diffusion on a curved surface coupled to diffusion in the volume: Application to cell biology, J. Comput. Phys., 226 (2007), pp. 1271-1290.

[31] E. A. Rakhmanov, E. B. Saff, and Y. M. Zhou, Minimal discrete energy on the sphere, Math. Res. Lett., 1 (1994), pp. 647-662.

[32] E. A. Rakhmanov, E. B. SAFF, And Y. M. Zhou, Electrons on the Sphere, in Computational Methods and Function Theory 1994 (Penang), Ser. Approx. Decompos. 5, World Scientific, River Edge, NJ, 1995, pp. 293-309.

[33] T. Ransford, Potential Theory in the Complex Plane, London Math. Soc. Stud. Texts 28, Cambridge University Press, Cambridge, UK, 1995.

[34] T. Ransford and J. Rostand, Computation of capacity, Math. Comp., 76 (2007), pp. 14991520.

[35] S. Redner, A Guide to First-Passage Time Processes, Cambridge University Press, Cambridge, UK, 2001.

[36] Z. Schuss, A. Singer, And D. Holcman, The narrow escape problem for diffusion in cellular microdomains, Proc. Natl. Acad. Sci. USA, 104 (2007), pp. 16098-16103.

[37] A. Singer, Z. Schuss, And D. Holcman, Narrow escape, Part III: Non-smooth domains and Riemann surfaces, J. Stat. Phys., 122 (2006), pp. 491-509.

[38] V. P. SMyshlyaev, The high-frequency diffraction of electromagnetic waves by cones of arbitrary cross sections, SIAM J. Appl. Math., 53 (1993), pp. 670-688.

[39] W. Stortelder, J. de Swart, and J. Pintér, Finding elliptic Fekete point sets: Two numerical solution approaches, J. Comput. Appl. Math., 130 (1998), pp. 205-216.

[40] R. Straube, M. J. Ward, And M. Falcke, Reaction-rate of small diffusing molecules on a cylindrical membrane, J. Stat. Phys., 129 (2007), pp. 377-405.

[41] R. Straube AND M. J. WARD, An asymptotic analysis of intracellular signaling gradients arising from multiple small compartments, SIAM J. Appl. Math., 70 (2009), pp. 248-269.

[42] A. Szabo, K. Schulten, and Z. Schulten, First passage time approach to diffusion-controlled reactions, J. Chem. Phys., 72 (1980), pp. 4350-4357.

[43] R. Szmytkowski, Closed form of the generalized Green's function for the Helmholtz operator on the two-dimensional unit sphere, J. Math. Phys., 47 (2006), 063506.

[44] A. Taflia And D. Holcman, Dwell time of a Brownian molecule in a microdomain with traps and a small hole on the boundary, J. Chem. Phys., 126 (2007), 234107.

[45] R. Varma, G. Campi, T. Yokosuka, T. Saito, and M. L. Dustin, T cell receptor-proximal signals are sustained in peripheral microclusters and terminated in the central supramolecular activation cluster, Immunity, 25 (2006), pp. 117-127.

[46] M. J. Ward, W. D. HenshaW, and J. B. Keller, Summing logarithmic expansions for singularly perturbed eigenvalue problems, SIAM J. Appl. Math., 53 (1993), pp. 799-828.

[47] D. Weaver, Diffusion-mediated localization on membrane surfaces, Biophys. J., 41 (1983), pp. $81-86$.

[48] L. L. Whyte, Unique arrangements of points on a sphere, Amer. Math. Monthly, 59 (1952), pp. 606-611.

[49] C. Wofsy, D. Coombs, And B. Goldstein, Calculations show substantial serial engagement of T cell receptors, Biophys. J., 80 (2001), pp. 606-612.

[50] T. Yokosuka, K. Sakata-Sogawa, W. Kobayashi, M. Hiroshima, A. Hashimoto-Tane, M. Tokunaga, And M. L. Dustin, Newly generated T cell receptor microclusters initiate and sustain $T$ cell activation by recruitment of Zap70 and SLP-76, Nat. Immunol., 6 (2005), pp. 1253-1262.

Copyright (c) by SIAM. Unauthorized reproduction of this article is prohibited. 